\title{
Bio-incrustantes marinos en el canal de Chacao, Chile: un estudio sobre potenciales interacciones con estructuras manufacturadas por el hombre
}

\author{
Marine bio-encrusting in the Chacao channel, Chile: a study of \\ potential interactions with man-made structures \\ Patricio H. Manríquez ${ }^{1}$, Eliseo Fica ${ }^{1}$, Verónica Ortiz \\ y Juan Carlos Castilla ${ }^{2,3}$
}

\begin{abstract}
'Laboratorio de Ecología y Conducta de la Ontogenia Temprana (LECOT), Centro de Estudios Avanzados en Zonas Áridas (CEAZA), Avenida Ossandón 877, Coquimbo, Chile. patriciohmanriquez@gmail.com

${ }^{2}$ Departamento de Ecología, Facultad de Ciencias Biológicas, Pontificia Universidad Católica de Chile, Casilla 114-D, Santiago, Chile

${ }^{3}$ Centro de Conservación Marina, Estación Costera de Investigaciones Marinas, Facultad de Ciencias Biológicas, Pontificia Universidad Católica de Chile, Casilla 114-D, Santiago, Chile
\end{abstract}

\begin{abstract}
The literature on marine bio-fouling (MBF) in Chile was revised and complemented with field data from the Chacao channel (Chiloé), identifying both the bio-fouling species associated with man-made structures (MMS) in the intertidal zone and species of algae, sessile and hemi-sessile invertebrates, present on natural substrata in the same area. In addition, to quantify settlement potential of the MBFA, artificial substrate plates (AS) were deployed in inter- and sub-tidal areas of the Chacao channel. The review identified 211 species, $85 \%$ of which were marine invertebrates and $15 \%$ macroalgae. The most representative taxa were arthropods, molluscs, annelids, which contributed $58 \%$ of the species of the Chilean MBF. The field data associated with MBF and with intertidal substrates indicated 133 species as potential components of the Chacao channel's MBF. On natural substrata and in decreasing order of abundance were recorded gastropod molluscs, crustaceans and seaweed. The most abundant species on MMS were molluscs, seaweeds and crustaceans. The species that settled on AS deployed in the intertidal zone were mussels, barnacles and macroalgae. In the subtidal zone the most abundant groups were macroalgae spores and bryozoans. Based on the literature review, the fished giant barnacle Austromegabalanus psittacus, the ascidian Pyura chilensis and the mussel Mytilus chilensis appear to be highly frequent and dominant species on subtidal natural hard-bottom. Because of their biological and ecological characteristics, these species have high degree of potential for colonising MMS deployed in the subtidal zone of the Chacao channel.
\end{abstract}

Key words: Bio-incrusting, Chacao channel, Chiloé, natural substrata, man-made substrata, Pyura chilensis, Austromegabalanus psittacus, Mytilus chilensis

\begin{abstract}
Resumen.- Se revisó literatura sobre los bio-incrustantes marinos (BIM) de Chile, complementado con campañas de terreno en el canal de Chacao (Chiloé), para registrar los BIM asociados a estructuras manufacturadas por el hombre (EMH) en la zona intermareal así como las especies de algas e invertebrados sésiles o hemisésiles presentes en la misma zona y potenciales BIM. Además, un tipo de sustrato artificial (SA) fue instalado en el inter y submareal del canal de Chacao para cuantificar el asentamiento de potenciales BIM. La revisión indicó una diversidad de 211 especies, correspondiendo 85\% a invertebrados y $15 \%$ a macroalgas. Los taxones más representativos fueron artrópodos, moluscos y anélidos, los cuales contribuyeron al 58\% de las especies de BIM registrados en Chile. La información de terreno indica 133 especies potencialmente BIM en el canal de Chacao. En sustratos naturales y en orden decreciente de abundancia se registraron los moluscos gastrópodos, crustáceos y macroalgas. En EMH del canal de Chacao los BIM más abundantes en orden decreciente fueron los moluscos, macroalgas y crustáceos. Sin embargo, los BIM más representados en SA desplegados en la zona intermareal fueron mitílidos, cirrípedos y macroalgas; mientras que en el submareal fueron esporas de macroalgas y briozoos. De los BIM recopilados desde la literatura, el cirrípedo Austromegabalanus psittacus, la ascidia Pyura chilensis y el mitílido Mytilus chilensis aparecen altamente frecuentes y dominantes en sustratos duros naturales, en especial en el canal de Chacao. Debido a sus características biológicas y ecológicas, estas especies destacan por su potencial para colonizar EHM en el submareal del canal de Chacao.
\end{abstract}

Palabras clave: Bio-incrustaciones, canal de Chacao, Chiloé, sustratos naturales, sustratos manufacturados por el hombre, Pyura chilensis, Austromegabalanus psittacus, Mytilus chilensis 


\section{INTRODUCCIÓN}

A nivel mundial se predice una expansión importante en el uso de fuentes de energías renovables limpias, entre ellas y relacionada con el mar destaca el desarrollo de la energía mareomotriz basada en las diferencias de las amplitudes de nivel de marea (Shaw et al. 2002, Falcão 2010, Langhamer et al. 2010). La implementación de dicha energía mareomotriz requiere el despliegue de estructuras manufacturadas por el hombre tales como turbinas mareomotrices y rotores que son posicionadas en el mar (Glasby \& Connell 1999, Bulleri \& Chapman 2010). Estos nuevos hábitats constituyen sustratos para el asentamiento y colonización/desarrollo de diversas especies marinas, que en su conjunto se denomina aquí como organismos bio-incrustantes marinos (fouling) (BIM). Así, el ensamble de bio-incrustantes marinos (EBIM) es definido como el conjunto de especies de invertebrados sésiles o hemisésiles y algas que se adhieren o se desplazan sobre sustratos artificiales manufacturados por el hombre y posicionados en el mar (Hillman 1977, Railkin 2004, nuestra definición). La mayoría de los organismos del EBIM se caracterizan por presentar altas tasas de reclutamiento y crecimiento, que los hace eficientes colonizadores de sustratos de origen antropogénico, entre cuyos principales grupos se destacan las algas, esponjas, hidrozoos, briozoos, moluscos, cirrípedos y ascidias (Richmond \& Seed 1991, Walker et al. 2007, Fitridge et al. 2012); caracterizándose algunas especies de mitílidos como Perumytilus purpuratus y Mytilus chilensis o de ascidias como Pyura chilensis y $P$. preaputialis como especies bio-ingenieras (Jones et. al. 1994, Castilla et al. 2004, Prado \& Castilla 2006, Sepúlveda et al. 2003). Durante la fase de dispersión, las larvas o esporas de estas especies son transportadas por las corrientes marinas y/o olas, se adhieren y crecen sobre sustratos artificiales como pilotes de muelles y puentes, boyas, defensas costeras, cascos de embarcaciones, redes de pesca, sistemas de cultivo, plataformas petroleras, ductos y turbinas submarinas (Richmond \& Seed 1991, Yan \& Yan 2003, Pacheco \& Garate 2005, Langhamer et al. 2009, Maar et al. 2009, Fitridge et al. 2012). En consecuencia, en la planificación del uso de estas estructuras en el mar (construcción, emplazamiento, servicio y eventual mantenimiento) requieren de estudios sobre los potenciales organismos bio-incrustantes del área de instalación del proyecto. El disponer de esta información permitirá predecir potenciales interferencias de los EBIM sobre las estructuras artificiales y planificar posibles medidas remediales, mitigación o de control. Los EBIM presentes sobre sustratos artificiales y aquellos ensambles presentes sobre sustratos naturales muchas veces difieren entre sí. Por ejemplo, los sustratos artificiales tienden a favorecer el asentamiento, reproducción y crecimiento de organismos filtradores como mitílidos, ascidias y cirrípedos (Glasby \& Connell 1999, Connell 2001, Svane \& Petersen 2001, Smith \& Rule 2002, Knott et al. 2004, Bulleri \& Airoldi 2005, Perkol-Finkel et al. 2006). Un conocimiento del potencial efecto de los EBIM sobre estructuras artificiales en el mar, previo a sus emplazamientos, requiere de información de largo plazo sobre las especies presentes en los substratos naturales del área de emplazamiento (i.e., inter y submareales), en sustratos artificiales similares en naturaleza a los que serán usados, e idealmente de las especies que se asientan en sustratos naturales y artificiales a diferentes niveles de profundidad.

En Chile, el canal de Chacao ( $\left.41^{\circ} \mathrm{S}\right)$ muestra amplitudes de nivel de marea de entre 5,5 y 6,0 m (Cáceres et al. 2003, Aiken 2008) y por ello constituye un sitio atractivo para la generación de energía mareomotriz (Hassan 2009). Desde el punto de vista ambiental, la puesta en marcha, funcionamiento y mantenimiento de recuperadores de energía mareomotriz requiere de información sobre los organismos potencialmente bio-incrustantes que están presentes en sustratos naturales y artificiales en torno al área a usar. Además, es conocido el efecto que ha tenido sobre la biota marina local el establecimiento mediado por la acción humana (i.e., aguas de lastres, cascos de embarcaciones, boyas) de especies no-nativas (Castilla et al. 2005). En Chile aún no existen recuperadores de energía mareomotriz instalados y por lo tanto no existe información sobre los EBIM que podrían interactuar con estas estructuras. Para el país, los registros y estudios de EBIM se relacionan principalmente a actividades de acuicultura, siendo los más numerosos aquellos estudios desarrollados en el norte de Chile (27-30 $\left.{ }^{\circ} \mathrm{S}\right)$, especialmente en asociación al cultivo de ostiones en las bahías Inglesa, La Herradura, Tongoy y Guanaqueros (Viviani \& DiSalvo 1980, Cañete \& Ambler 1990, Ambler \& Cañete 1991, Basilio et al. 1995, Uribe et al. 2001). Estos estudios se realizaron para conocer los organismos bio-incrustantes e implementar mecanismos de mitigación y control de su asentamiento en los sistemas de cultivo. Inexplicablemente, en el extremo sur de Chile, donde se concentra la inmensa mayoría de la actividad de acuicultura en el país (SERNAPESCA 2010), los estudios publicados para EBIM son muy escasos, destacándose 
el de Von Plessing (1981) en relación con cultivos de mitílidos en la bahía de Yaldad $\left(43^{\circ} \mathrm{S}\right)$.

En este trabajo el área de estudio se focalizó en la zona del canal de Chacao, Chiloé, zona en la cual se desarrollan estudios preliminares y exploratorios para la futura instalación submarina de dispositivos recuperadores de energía mareomotriz (Proyecto FONDEF D09I1052)․․ El objetivo general de este trabajo fue identificar los organismos EBIM sésiles y hemisésiles, presentes en sustratos duros naturales y en sustratos artificiales en la zona del canal de Chacao, complementando con información biológica y pesquera existente sobre especies bio-incrustantes abundantes, con crecimiento rápido y biomasas elevadas. Los objetivos específicos fueron: (1) revisar la información publicada sobre los EBIM registrados en la costa de Chile y en especial para la zona de interés en Chiloé y el canal de Chacao, (2) recoger información in situ de las especies de invertebrados (i.e., con tamaños corporales $>0,5 \mathrm{~mm}$ y visibles a ojo desnudo) sésiles y hemisésiles así como las algas para conocer los potenciales bio-incrustantes presentes en sustratos duros naturales y artificiales; (3) evaluar el asentamiento en un tipo de sustrato artificial desplegado en la zona inter y submareal para conocer los potenciales bio-incrustantes presentes.

\section{MATERIALES Y MÉTODOS}

ReVisión de literatura SOBRe organismos bioinCrustantes en las costas de ChILE

La información referente a la composición del ensamble de especies bio-incrustantes registradas para la costa de Chile y en particular para la zona de estudio en el canal de Chacao, fue recopilada de la literatura publicada en revistas de corriente principal y en tesis de grado en temas afines.

Organismos de LA ZONA INTERMAREAL DE LA COSTA DEL Canal de Chacao que son potenciales bioINCRUSTANTES EN SUSTRATOS NATURALES

Para describir la composición de especies de invertebrados sésiles o hemisésiles y algas presentes en la zona intermareal rocosa en las inmediaciones de la zona de interés (potenciales EBIM), se realizaron inventarios biológicos sistemáticos en 6 localidades (Fig. 1), durante 6 mareas bajas de sizigia de marzo y abril de 2012 con alturas promedio $( \pm \mathrm{EE})$ de $1,27 \mathrm{~m}( \pm 0,41)$. Durante cada inventario las observaciones se realizaron en forma simultánea e independiente por 4 observadores en movimiento en 4 sectores diferentes. Cada observador registró en 6 series consecutivas de 5 min cada una (i.e., esfuerzo total de muestreo de $30 \mathrm{~min}$ ) a nivel de especies los invertebrados sésiles o hemisésiles y algas presentes en la superficie y oquedades de sustratos intermareales primarios durante el tránsito sostenido del observador a lo largo de la franja alta, media y baja intermareal rocosa, caracterizadas por especies indicadoras, como líquenes, litorínidos, cirrípedos, mitílidos y macroalgas (Castilla 1981, 2008). Durante el esfuerzo total de muestreo (30 min), cada observador recorrió las 3 franjas intermareales y una distancia de $5 \mathrm{~m}$ fue mantenida entre los sectores cubiertos por cada observador. Además, las observaciones incluyeron registros sobre la superficie inferior de rocas de mediano tamaño (menos de $5 \mathrm{~kg}$ de peso) posibles de voltear por el observador. Cuando no fue posible la identificación in situ de las especies, los ejemplares fueron removidos y almacenados en bolsas plásticas rotuladas para su posterior identificación en el laboratorio. Muchos estudios ecológicos requieren estimaciones de la riqueza de especie de un área determinada y para ello se requiere la elaboración de listados lo más completos posible sobre la presenciaausencia de especies. La relación entre el tiempo de colección u observación (i.e., esfuerzo) y el número acumulado de especies se denominan curvas de acumulación de especies (Soberón \& Llorente 1993, Thompson et al. 2003). Estas curvas son un método simple, uniforme y sin sesgo para estimar la riqueza de especies de un hábitat en particular. En el presente estudio el número promedio acumulado de especies por localidad de estudio fue expresado como función del esfuerzo de muestreo para generar curvas de saturación de riqueza de especies (Ugland et al. 2003). Esto permitió estimar la riqueza de especies en cada una de las zonas, en función de la estabilización del número acumulado de especies, independientemente de los incrementos en el esfuerzo de muestreo. 


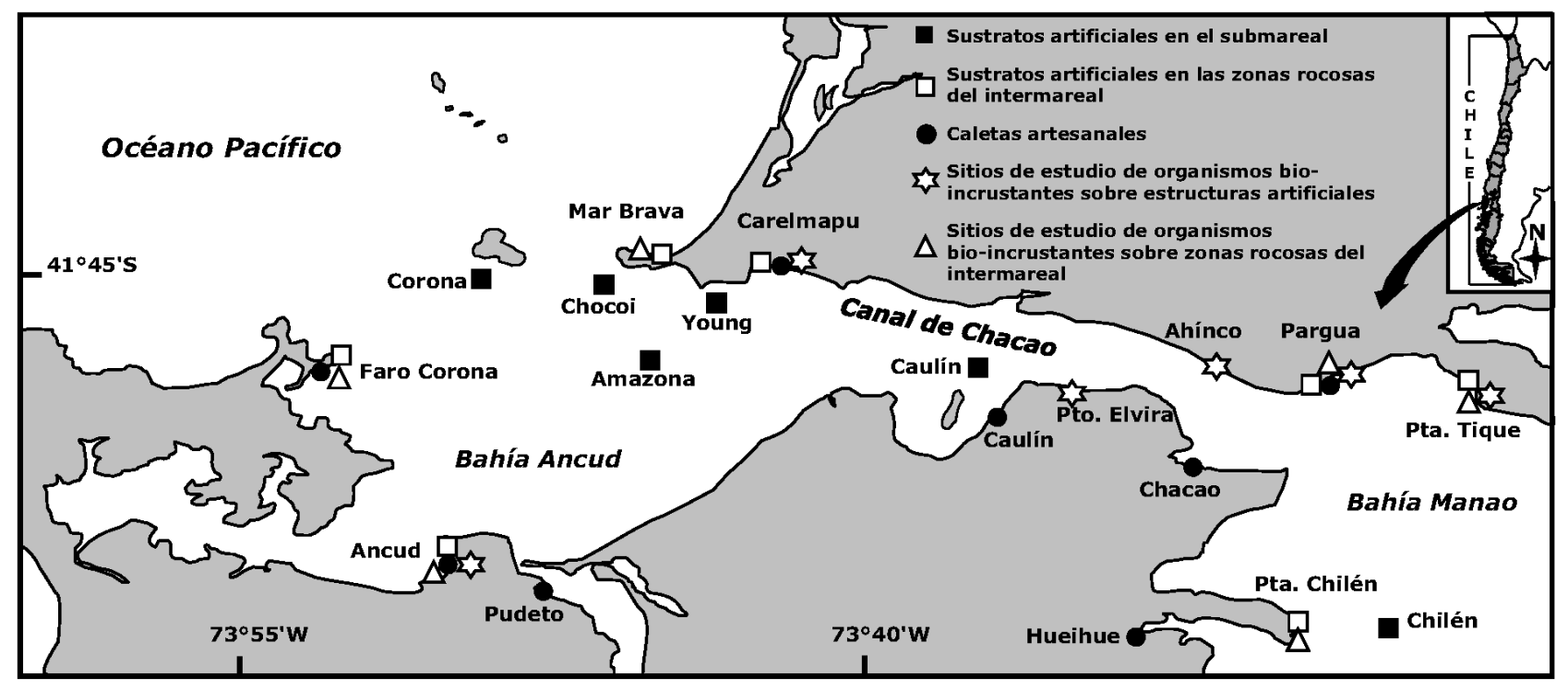

Figura 1. Mapa de la zona de estudio en el canal de Chacao. Se indica la ubicación de los sitios de estudio en los cuales se realizaron observaciones de los organismos bio-incrustantes presentes sobre la superficie de estructuras manufacturadas por el hombre y localizadas en las zonas rocosas intermareales y observaciones sobre la superficie de las zonas rocosas intermareales. Además, se señalan las caletas de pescadores artesanales y los sitios de estudio donde se instalaron sustratos artificiales en las zonas rocosas intermareales y submareales / Map of the study zone at the Chacao channel. In the map are depicted: the locations of the sampling sites were the observations of the bio-encrusting organisms present on intertidal man-made structures and on the surface of rocky intertidal habitats were conducted. Moreover, the location of the artisanal fisher coves and the study sites were the artificial substrates were placed in the rocky intertidal and subtidal are also depicted

\section{Organismos bio-incrustantes en Sustratos MANUFACTURADOS POR EL HOMBRE EN LA ZONA INTERMareal de LA COSTA DEL CANAL DE CHACAO}

Para describir la composición de especies de invertebrados sésiles o hemisésiles y algas presentes en sustratos manufacturados por el hombre en las inmediaciones de la zona de interés se realizaron registros visuales en 6 localidades en la zona de estudio (Fig. 1), durante 6 mareas de sizigia de enero y marzo 2012 con alturas promedio de $0,62 \mathrm{~m}( \pm 0,16)$, e incluyeron pilotes de variada naturaleza y defensas costeras de concreto. Los registros de especies fueron realizados por 2 (enero) y 4 (marzo) observadores. Al igual que en el muestreo en el intermareal rocoso, los registros en sustratos artificiales incluyeron la identificación in situ hasta nivel de especie. En el caso de las defensas costeras cada observador realizó las observaciones en forma independiente sobre un sector de la superficie total de los sustratos analizados. Sin embargo, debido a la reducida superficie asociada a los pilotes las observaciones en este tipo de sustrato fueron realizadas por cada observador sobre las mismas estructuras. Independiente del tipo de sustrato las observaciones de cada observador fueron realizadas durante $30 \mathrm{~min}$. Cuando no fue posible realizar la identificación de las especies, esta fue realizada en el laboratorio en individuos removidos y almacenados en bolsas rotuladas.

\section{ASENTAMIENTo DE ORGANISMOS BIO-INCRUSTANTES EN LA zona intermareal del Canal de Chacao}

El tipo de bio-incrustantes y la magnitud de su asentamiento en la zona intermareal rocosa se estudiaron utilizando como proximal placas de acrílico de amplio uso en la literatura (Navarrete et al. 2002), de $5 \mathrm{~cm}$ de ancho, 5 $\mathrm{cm}$ de alto y $0,5 \mathrm{~cm}$ de grosor, para aumentar la heterogeneidad de la superficie del acrílico la superficie superior de las placas fue cubierta con cinta antideslizante $\left(3 \mathrm{M}^{\circledR}\right)$. Las placas fueron desplegadas en la zona intermareal alta y baja en sectores expuestos al oleaje por un periodo aproximado de 3 meses en 3 localidades de la zona de estudio: 1 en el borde continental y 2 en el borde insular del canal de Chacao (Fig. 1). Las placas fueron adheridas a las rocas con un perno inoxidable fijado en una perforación y con un tarugo plástico (Anexo 1). El número de placas por nivel, localidad y tiempo que estas fueron expuestas en el ambiente se detallan en la Tabla 1. 
Tabla 1. Fechas de instalación y retiro de las placas experimentales instaladas en zonas inter y submareales para registrar el asentamiento de organismos potencialmente bio-incrustantes en las localidades de estudio en el canal de Chacao. Se incluyen nombres y coordenadas de cada localidad / Dates of installation and removal of the experimental plates installed in inter and subtidal areas to record the settlement of potentially bio-fouling organisms in the studied localities at the Chacao channel. The names and coordinates of each site are included

\begin{tabular}{|c|c|c|c|c|c|c|c|}
\hline Localidad & Zona & $\begin{array}{l}\text { Profundidad } \\
\text { (m) }\end{array}$ & $\begin{array}{l}\text { Ubicación } \\
\text { geográfica }\end{array}$ & $\begin{array}{l}\text { Fecha de } \\
\text { instalación }\end{array}$ & $\begin{array}{c}\text { Fecha de } \\
\text { retiro }\end{array}$ & $\begin{array}{l}\text { Días de } \\
\text { exposición }\end{array}$ & $\begin{array}{l}\mathrm{N}^{\circ} \mathrm{de} \\
\text { placas }\end{array}$ \\
\hline Punta Tique (C) & Intermareal & - & $\begin{array}{l}41^{\circ} 32^{\prime} 14^{\prime \prime S} \\
73^{\circ} 17^{\prime} 07^{\prime \prime} \mathrm{W}\end{array}$ & $06 / 03 / 2012$ & $23 / 06 / 2012$ & 109 & $\begin{array}{l}10(\mathrm{~S}) \\
10(\mathrm{I})\end{array}$ \\
\hline Punta Chilén (I) & Intermareal & - & $\begin{array}{l}42^{\circ} 29^{\prime} 05^{\prime \prime S} \\
73^{\circ} 13^{\prime} 40^{\prime \prime} \mathrm{W}\end{array}$ & $16 / 03 / 2012$ & $25 / 06 / 2012$ & 101 & $\begin{array}{l}10(\mathrm{~S}) \\
10(\mathrm{I})\end{array}$ \\
\hline Faro Corona (I) & Intermareal & - & $\begin{array}{l}41^{\circ} 47^{\prime} 06^{\prime \prime} \mathrm{S} \\
73^{\circ} 53^{\prime} 18^{\prime \prime} \mathrm{W}\end{array}$ & $18 / 03 / 2012$ & $24 / 06 / 2012$ & 98 & $\begin{array}{l}10(\mathrm{~S}) \\
10(\mathrm{I})\end{array}$ \\
\hline Corona & Submareal & 32 & $\begin{array}{l}41^{\circ} 45^{\prime} 45^{\prime \prime} \mathrm{S} \\
73^{\circ} 49^{\prime} 59^{\prime \prime} \mathrm{W}\end{array}$ & $21 / 05 / 2012$ & $8 / 07 / 2012$ & 48 & 12 \\
\hline Chocoi & Submareal & 34 & $\begin{array}{l}41^{\circ} 45^{\prime} 17^{\prime \prime} \mathrm{S} \\
73^{\circ} 46^{\prime} 27^{\prime \prime} \mathrm{W}\end{array}$ & $22 / 05 / 2012$ & $11 / 07 / 2012$ & 50 & 12 \\
\hline Amazonas & Submareal & 27 & $\begin{array}{l}41^{\circ} 46^{\prime} 51^{\prime \prime S} \\
73^{\circ} 45^{\prime} 28^{\prime \prime} \mathrm{W}\end{array}$ & $23 / 05 / 2012$ & $10 / 07 / 2012$ & 48 & 12 \\
\hline Young & Submareal & 37 & $\begin{array}{l}41^{\circ} 45^{\prime} 43^{\prime \prime} \mathrm{S} \\
73^{\circ} 41^{\prime} 11^{\prime \prime} \mathrm{W}\end{array}$ & $24 / 05 / 2012$ & $9 / 07 / 2012$ & 46 & 12 \\
\hline Caulin & Submareal & 17 & $\begin{array}{l}41^{\circ} 47^{\prime} 11^{\prime \prime S} \\
73^{\circ} 36^{\prime} 29^{\prime \prime} \mathrm{W}\end{array}$ & $20 / 05 / 2012$ & $6 / 07 / 2012$ & 47 & 12 \\
\hline Chilén & Submareal & 35 & $\begin{array}{l}41^{\circ} 53^{\prime} 49^{\prime \prime} \mathrm{S} \\
73^{\circ} 24^{\prime} 46^{\prime \prime} \mathrm{W}\end{array}$ & $25 / 05 / 2012$ & $6 / 07 / 2012$ & 42 & 12 \\
\hline
\end{tabular}

En la zona intermareal las placas fueron desplegadas en la parte superior (S) o inferior (I) de las zonas rocosas de localidades en el borde continental (C) o insular (I)

Luego de expuestas las placas fueron retiradas (periodos variables con un mínimo de 1,5 meses) y almacenadas en bolsas plásticas individuales rotuladas, para posteriormente identificar y cuantificar en el laboratorio las especies.

\section{ASENTAMIENTO DE ORGANISMOS BIO-INCRUSTANTES EN LA zona submareal del canal de Chacao}

En forma similar a lo descrito anteriormente, las especies de organismos bio-incrustantes y la magnitud de su asentamiento en la zona submareal se estudiaron a través del uso de placas de acrílico, como ya descritas. Las placas se adhirieron con masilla epóxica submarina (Sherwin Williams $\left.{ }^{\circledR}\right)$, a bloques de concreto anclados en el fondo de cada localidad. En cada una de las 6 localidades (Fig. 1), los bloques con 12 placas (3 por cada cara del bloque, Anexo 1) fueron depositados (mayo, 2012) a profundidades entre 17 y $37 \mathrm{~m}$, a una distancia no mayor a los $50 \mathrm{~m}$ de la costa (Tabla 1). En todas las localidades los bloques se depositaron en fondos arenosos y luego de 6 a 7 semanas éstos fueron izados para remover las placas. Las placas fueron almacenadas y analizadas en el laboratorio para identificar y cuantificar las especies asentadas. Tal como se ha descrito en trabajos similares utilizando este mismo tipo de placas (Navarrete et al. 2002), tanto para las placas desplegadas en la zona intermareal como en la submareal la abundancia de los bio-incrustantes fue estandarizada al número de ejemplares asentados por $100 \mathrm{~cm}^{2}$ por día.

\section{BIO-INCRUSTANTES CON MAYOR BIOMASA EN LA ZONA SubMareal de la costa del CaNal de ChacaO}

Estudios previos han determinado que altos valores de biomasa de EBIM en sustratos experimentales se asocian con especies bio-incrustantes como cirrípedos, ascidias y mitílidos (Rajagopal et al. 1990, Cao et al. 2013). En Chile, debido a sus altas tasas de crecimiento, 3 especies de invertebrados se vislumbran como importantes candidatos a constituirse en EBIM: la ascidia Pyura chilensis (piure), el cirrípedo gigante Austromegabalanus psittacus (picoroco) y el mitílido Mytilus chilensis (chorito). Las 2 primeras especies han sido explotadas desde bancos naturales en la zona de estudio. Sin embargo, M. chilensis es una especie ampliamente 
cultivada en la misma zona. Además, al ser estas especies bio-ingenieras (Sepúlveda et al. 2003, Carranza et al. 2009) su presencia sobre estructuras manufacturadas por el hombre se asociará, necesariamente al asentamiento y establecimiento de otras especies de invertebrados y algas.

\section{Resultados}

\section{REVISIÓN SOBRE ORGANISMOS BIO-INCRUSTANTES EN LAS costas de ChiLe}

La información recopilada indica que para Chile se han registrado 211 especies/taxones de bio-incrustantes (Anexo 2). Estas comprenden 32 especies de algas y 179 invertebrados marinos. Del total de las especies registradas sólo $6(2,84 \%)$ corresponden a especies introducidas: las ascidias Ciona intestinalis, Asterocarpa humilis y Molgula ficus, el alga Chlorophyta Codium fragile y colonias de los briozoos arborescentes Bugula flabellata y $B$. neritina. Los taxones más representados corresponden a Arthropoda, Mollusca y Annelida (Tabla 2). En el presente estudio en el canal de Chacao sobre

Tabla 2. Contribución absoluta (número de especies) y porcentual de los diferentes taxones de invertebrados sésiles o hemisésiles y macroalgas que constituyen el ensamble de bio-incrustantes marinos descritos por la literatura para toda la costa de Chile / Absolute contribution (number of species) and percentage of different taxa of sessile or hemisessile invertebrates and macroalgae constituting the marine bio-fouling assembly described in the literature for the entire coast of Chile

\begin{tabular}{lcc}
\hline Fila / División & Especies & Porcentaje \\
\hline Chlorophyta & 5 & 2,37 \\
Phaeophyta & 14 & 6,64 \\
Rhodophyta & 13 & 6,16 \\
Porifera & 4 & 1,90 \\
Cnidaria & 17 & 8,06 \\
Platyhelminthes & 5 & 2,37 \\
Nemertina & 3 & 1,42 \\
Annelida & 31 & 14,69 \\
Arthropoda & 53 & 25,12 \\
Mollusca & 37 & 17,54 \\
Echinodermata & 3 & 1,42 \\
Urochordata & 15 & 7,11 \\
Ectoprocta & 11 & 5,21 \\
TOTAL & 211 & 100 \\
\hline
\end{tabular}

diversos sustratos manufacturados por el hombre se registraron 32 especies de bio-incrustantes (Tabla 3 ). Veinticinco de estas especies corresponden a nuevos registros de especies bio-incrustantes para Chile. En consecuencia, considerando lo compilado en la literatura (Anexo 2) y lo registrado en este estudio (Tabla 3) el número de especies bio-incrustantes que potencialmente se podrían registrar sobre la superficie de estructuras manufacturadas por el hombre en la costa de Chile alcanza a 236 especies.

Organismos de la ZONA INTERMAREAL DE LA COSTA DEL Canal de Chacao que son potenciales bioINCRUSTANTES EN SUSTRATOS NATURALES

Las curvas de saturación de especies de invertebrados sésiles o hemisésiles y algas generadas a partir de las observaciones realizadas en la zona intermareal rocosa de la costa continental e insular del canal de Chacao (Fig. 1), indican que $30 \mathrm{~min}$ de observación son suficientes para alcanzar la saturación del número de especies en las 3 localidades del borde continental del canal de Chacao. En estas localidades (con el método usado) la riqueza de especies fluctuó entre 27 y 34. Sin embargo, para el borde insular la curva de saturación sólo alcanzó valores asintóticos para la localidad de Punta Chilén (Fig. 2a). En esta localidad la riqueza promedio de especies sésiles o hemisésiles fue 37. Para Fuerte de San Antonio y Faro Corona el número promedio máximo de especies alcanzó valores de 31 y 42, respectivamente (Fig. 2b). Las especies registradas en los muestreos de la zona intermareal de ambos bordes del canal de Chacao destacan 33 especies de macroalgas y 100 especies de invertebrados sésiles o hemisésiles (Anexo 3).

\section{Organismos bio-incrustantes en Sustratos MANUFACTURADOS POR EL HOMBRE EN LA ZONA INTERMareal de la COSTa del CaNal de ChacaO}

Los sustratos artificiales analizados fueron defensas costeras de concreto, pilotes de madera, pilotes de concreto-arena y pilotes de concreto-hierro (Tabla 3). Las especies de algas bio-incrustantes más frecuentes en estos sustratos pertenecen a Rhodophyta y Chlorophyta. Las especies de invertebrados sésiles o hemisésiles más frecuentes en estos sustratos fueron especies de Mollusca, Arthropoda, Cnidaria y Echinodermata (Tabla 3 ). En total 7 especies de algas y 25 de invertebrados fueron registrados sobre estos sustratos (Tabla 3). Todas las especies de bio-incrustantes registradas en estos sustratos también fueron registradas en zonas rocosas naturales de la zona intermareal. 
Tabla 3. Especies bio-incrustantes $(n=32)$ registradas en sustratos manufacturados por el hombre y ubicados en la zona intermareal del canal de Chacao. Se indica el tipo de sustrato donde fueron realizados los registros de las especies (i.e., defensas costeras de concreto, pilotes de madera, pilotes mixtos concreto-arena y pilotes mixtos concreto-hierro), el grupo taxonómico, si corresponde a una especie de invertebrado sésil (S) o hemisésil (H) en su fase post-larval y si es una especie nativa (N) o introducida (I) / Bio-fouling species $(n=32)$ recorded on man-made substrates and located in the intertidal zone the Chacao channel. The type of substrate were the records were made (i.e., concrete coastal defenses, wood piles, sandconcrete piles and concrete-iron piles), the taxonomic group, if the invertebrate were sessile (S) or hemisessile species $(\mathrm{H})$ in their post-larval phase and if they were native $(\mathrm{N})$ or introduced species $(\mathrm{I})$ are indicated

\begin{tabular}{|c|c|c|c|c|}
\hline Filum/División & Nombre científico & $\mathrm{H} / \mathrm{S}$ & $\begin{array}{l}\mathrm{N} / \mathrm{I} \\
(*)\end{array}$ & $\begin{array}{l}\text { Tipo de sustrato } \\
\text { artificial }(* *)\end{array}$ \\
\hline \multirow[t]{2}{*}{ Chlorophyta } & Rama novaezelandiae $\dagger$ & S & $\mathrm{N}$ & $\mathrm{DC} / \mathrm{PM} / \mathrm{PCA}$ \\
\hline & Ulva compressa & $\mathrm{S}$ & $\mathrm{N}$ & $\mathrm{DC} / \mathrm{PM} / \mathrm{PCA}$ \\
\hline \multirow[t]{5}{*}{ Rhodophyta } & Gelidium pusillum $\dagger$ & $\mathrm{S}$ & $\mathrm{N}$ & DC/PCA \\
\hline & Hildenbrandia sp. $\dagger$ & $\mathrm{S}$ & - & $\mathrm{DC}$ \\
\hline & Mazzaella laminarioides $\dagger$ & $\mathrm{S}$ & $\mathrm{N}$ & $\mathrm{DC}$ \\
\hline & Nothogenia fastigiata $\dagger$ & $\mathrm{S}$ & $\mathrm{N}$ & $\mathrm{DC}$ \\
\hline & Porphyra columbinat & S & $\mathrm{N}$ & $\mathrm{DC}$ \\
\hline \multirow[t]{3}{*}{ Cnidaria } & Acontiaria sp. $\dagger$ & $\mathrm{S}$ & - & $\mathrm{DC}$ \\
\hline & Anthopleura hermafroditica $\dagger$ & $\mathrm{S}$ & $\mathrm{N}$ & $\mathrm{DC}$ \\
\hline & Phymactis clematis & $\mathrm{S}$ & $\mathrm{N}$ & $\mathrm{DC}$ \\
\hline \multirow[t]{3}{*}{ Mollusca: Bivalvia } & Mytilus chilensis & $\mathrm{S}$ & $\mathrm{N}$ & $\mathrm{DC}$ \\
\hline & Ostrea chilensis† & $\mathrm{S}$ & $\mathrm{N}$ & $\mathrm{DC}$ \\
\hline & Perumytilus purpuratus $\dagger$ & $\mathrm{S}$ & $\mathrm{N}$ & DC \\
\hline \multirow[t]{11}{*}{ Mollusca: Gastropoda } & Acanthina monodon† & $\mathrm{H}$ & $\mathrm{N}$ & $\mathrm{DC}$ \\
\hline & Austrolittorina araucana $\dagger$ & $\mathrm{H}$ & $\mathrm{N}$ & $\mathrm{DC}$ \\
\hline & Concholepas concholepas & $\mathrm{H}$ & $\mathrm{N}$ & $\mathrm{DC}$ \\
\hline & Diloma nigerrima $\dagger$ & $\mathrm{H}$ & $\mathrm{N}$ & $\mathrm{DC}$ \\
\hline & Nacella deaurata $\dagger$ & $\mathrm{H}$ & $\mathrm{N}$ & $\mathrm{DC}$ \\
\hline & Nacella magellanica $\dagger$ & $\mathrm{H}$ & $\mathrm{N}$ & $\mathrm{DC}$ \\
\hline & Prisogaster niger & $\mathrm{H}$ & $\mathrm{N}$ & $\mathrm{DC}$ \\
\hline & Scurria araucana† & $\mathrm{H}$ & $\mathrm{N}$ & $\mathrm{DC}$ \\
\hline & Scurria cecilianat & $\mathrm{H}$ & $\mathrm{N}$ & $\mathrm{DC}$ \\
\hline & Siphonaria lessoni & $\mathrm{H}$ & $\mathrm{N}$ & $\mathrm{DC}$ \\
\hline & Tegula atra† & $\mathrm{H}$ & $\mathrm{N}$ & $\mathrm{DC}$ \\
\hline \multirow[t]{2}{*}{ Mollusca: Polyplacophora } & Chiton granosus $\dagger$ & $\mathrm{H}$ & $\mathrm{N}$ & $\mathrm{DC}$ \\
\hline & Chiton magnificus $\dagger$ & $\mathrm{H}$ & $\mathrm{N}$ & $\mathrm{DC}$ \\
\hline \multirow[t]{5}{*}{ Arthropoda: Crustacea } & Amphipoda spp. & $\mathrm{H}$ & - & $\mathrm{DC}$ \\
\hline & Isopoda spp. & $\mathrm{H}$ & - & $\mathrm{DC}$ \\
\hline & Jehlius cirratus $\dagger$ & $\mathrm{S}$ & $\mathrm{N}$ & $\mathrm{DC} / \mathrm{PM} / \mathrm{PCA} / \mathrm{PCF}$ \\
\hline & Notochthamalus scabrosus $\dagger$ & $\mathrm{S}$ & $\mathrm{N}$ & $\mathrm{DC} / \mathrm{PM} / \mathrm{PCA} / \mathrm{PCF}$ \\
\hline & Petrolisthes violaceus $\dagger$ & $\mathrm{H}$ & $\mathrm{N}$ & $\mathrm{DC}$ \\
\hline Echinodermata & Loxechinus albus $\dagger$ & $\mathrm{H}$ & $\mathrm{N}$ & $\mathrm{DC}$ \\
\hline
\end{tabular}

-: Ausencia de información, si la especie es nativa o introducida.

**DC: Defensa costera de concreto $\left(\mathrm{N}=2 ; 20\right.$ a $\left.40 \mathrm{~m}^{2}\right)$, PM: Pilote madera $\left(\mathrm{N}=6 ; 0,6\right.$ a $\left.1 \mathrm{~m}^{2}\right)$,

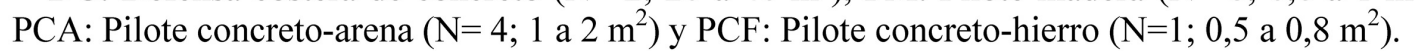

$\dagger$ : Indica a la especie como nuevo registro de bio-incrustante para Chile 


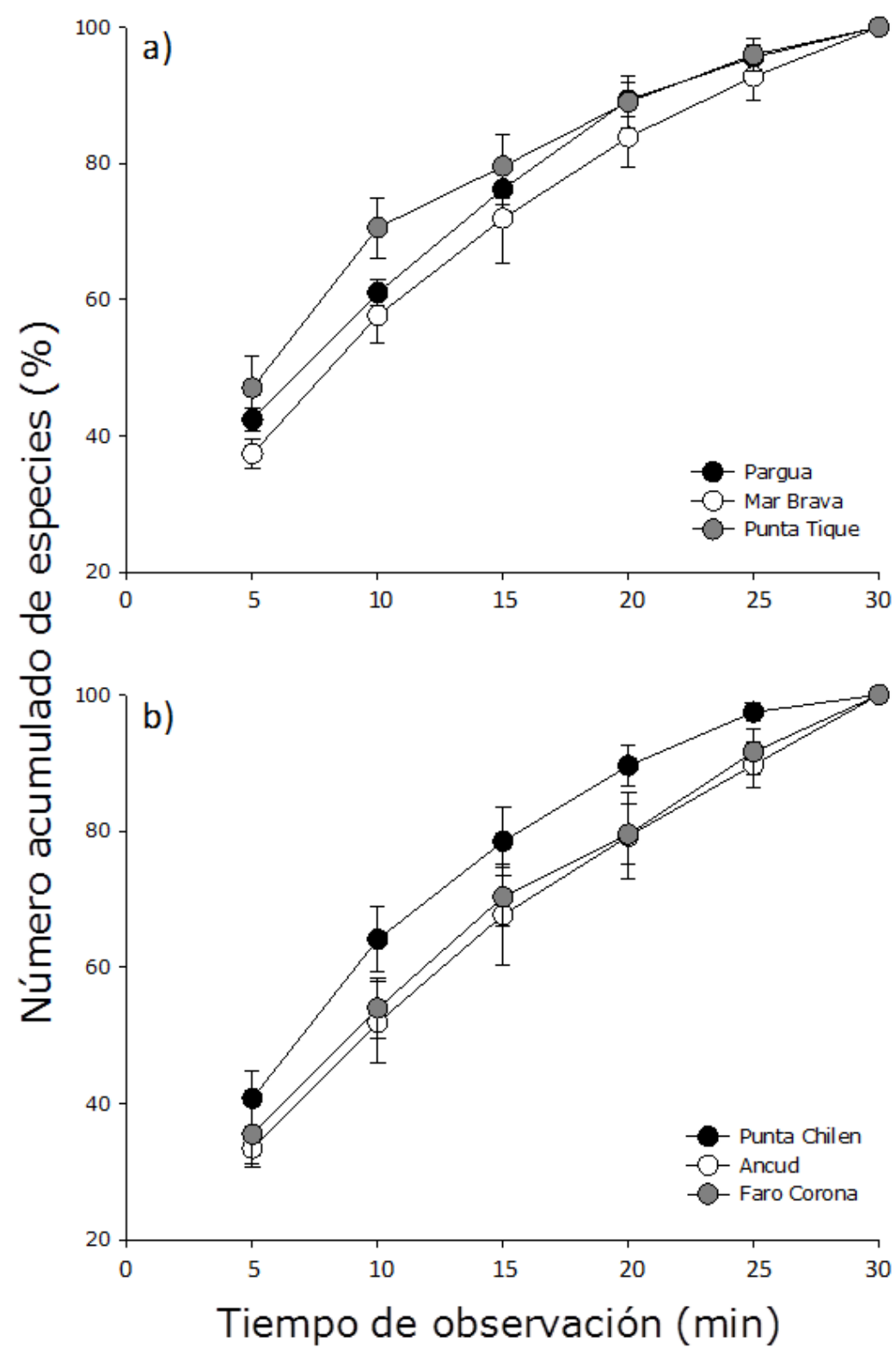

Figura 2. Curvas de saturación de especie (porcentaje promedio $\pm \mathrm{EE}$ ) de invertebrados y macroalgas elaboradas a partir de los registros hechos por 4 observadores en 6 series consecutivas de $5 \mathrm{~min}$ en cada localidad de estudio. Se indican localidades ubicadas en el borde continental: a) Mar Brava, Punta Tique y Pargua; y en el borde insular: b) Punta Chilén, Ancud y Faro Corona del canal de Chacao / Saturation species curves (average percentage \pm SE) of invertebrates and macroalgae build from records made by 4 observers in 6 consecutive series of 5 min in each study locality. Localities located in the continental border: a) Mar Brava, Punta Tique and of Pargua and insular border: b) Punta Chilén, Ancud and Faro Corona) of the Chacao channel 


\section{ASENTAMIENTo De ORGANiSMos BiO-INCRUSTANTES EN LA zona intermareal del Canal de ChacaO}

Los principales invertebrados sésiles o hemisésiles que se registraron sobre la superficie de las placas de acrílico, en las 3 localidades investigadas, pertenecen a grupos de Mollusca (Bivalvia) y Arthropoda (Crustacea), por otra parte los principales taxones de algas corresponden a esporas de Rhodophyta y Chlorophyta (Tabla 4).

\section{AsENTAMIENTo de ORganismos BiO-INCRUSTANTES EN LA zona submareal del Canal de ChacaO}

Los principales taxones de invertebrados sésiles o hemisésiles que se registraron sobre la superficie de las placas de asentamiento submareal en las 6 localidades investigadas pertenecen a Mollusca (Bivalvia), Arthropoda (Crustacea) y Bryozoa, mientras que los principales taxones de algas registradas fueron esporas de Rhodophyta, Phaeophyta y Chlorophyta (Tabla 5). Debido a que la totalidad de los registros de bioincrustantes sobre las placas de acrílico submareales correspondió a estadios tempranos de su ontogenia, no fue posible realizar la identificación de las especies.

\section{BIO-INCRUSTANTES CON MAYOR BIOMASA EN LA ZONA Submareal del Canal de Chacao}

Individuos de la ascidia $P$. chilensis y del cirripedio $A$. psittacus habitan desde la zona intermareal baja hasta el submareal de la costa de Perú y Chile (Vásquez 1983, López 1994). En Chile las poblaciones de P. chilensis se extienden hasta la isla de Chiloé, y corresponde a una ascidia individual, de gran tamaño que en el ambiente submareal puede llegar a formar extensas agregaciones de varios metros cuadrados formadas por cientos de ejemplares (Davis 1995). Austromegabalanus psittacus es el cirrípedo no pedunculado más grande del mundo (Darwin 1854), y a menudo forma agrupaciones de hasta 1,000 ind $\mathrm{m}^{2}$ (López et al. 2007). Con fines de hacer posible la acuicultura de esta especie se ha registrado que su asentamiento puede ocurrir en diversos tipos de sustratos artificiales (López et al. 2012). Además, luego de ocurrido el asentamiento y crecimiento de $A$. psittacus cada metro de línea de cultivo puede producir en promedio entre 8 a 15 $\mathrm{kg}$ de biomasa lo que corresponde en promedio a 100 ejemplares de tamaño comercial (López et al. 2012). En consecuencia, es esperable que ambas especies se puedan asentar y crecer sobre estructuras manufacturadas por el hombre y desplegadas en el submareal. Debido al tamaño, y lo gregario de estas especies, es altamente probable que su asentamiento sobre estructuras artificiales derive en incrementos importantes del peso de estas y la obstrucción de su funcionamiento óptimo. Una interacción similar se espera que ocurra entre estas estructuras y ejemplares del mitílido $M$. chilensis, especie abundante en los centros de cultivo presentes en la zona de estudio (SERNAPESCA 2010), que también se registra a lo largo de gran parte de la costa de Chile en forma de bancos naturales, desde la zona intermareal hasta profundidades de 25 m (Brattström \& Johanssen 1983, Lancellotti \& Vázquez 2000). La información disponible desde el 2000

Tabla 4. Abundancia (ind $100 \mathrm{~cm}^{-2} \mathrm{dia}^{-1}$ ) y cobertura (\%) promedio ( $\pm \mathrm{EE}$ ) de invertebrados marinos y algas asentadas por unidad de superficie y tiempo sobre la superficie de sustratos artificiales desplegados en la zona intermareal rocosa de 6 sitios de estudio en el canal de Chacao / Mean ( \pm SE) abundance (ind $100 \mathrm{~cm}^{-2}$ day $^{-1}$ ) and coverage (\%) of settled marine invertebrates and algae per unit of area and time recorded on the surface of artificial substrates deployed in the rocky intertidal zone of 6 study sites at the Chacao channel

\begin{tabular}{|c|c|c|c|c|c|c|c|}
\hline \multirow[b]{3}{*}{ Nivel mareal } & \multirow[b]{3}{*}{ Especie } & \multirow{2}{*}{\multicolumn{2}{|c|}{$\begin{array}{c}\text { Borde Continental } \\
\text { Punta Tique }\end{array}$}} & \multicolumn{4}{|c|}{ Borde Insular } \\
\hline & & & & \multicolumn{2}{|c|}{ Punta Chilén } & \multicolumn{2}{|c|}{ Faro Corona } \\
\hline & & Abundancia & Cobertura & Abundancia & Cobertura & Abundancia & Cobertura \\
\hline \multirow[t]{4}{*}{ Superior } & Chlorophyta & - & - & - & - & - & $27,78(3,29)$ \\
\hline & Jehlius cirratus & - & - & $0,008(0,005)$ & - & - & - \\
\hline & Mytilidae & - & - & $0,012(0,006)$ & - & - & - \\
\hline & Porphyra columbina & - & - & - & - & - & $3,80(2,81)$ \\
\hline \multirow[t]{5}{*}{ Inferior } & Chlorophyta & - & $1,41(0,39)$ & - & $1,60(0,66)$ & - & $13,83(4,37)$ \\
\hline & Jehlius cirratus & - & - & $0,005(0,004)$ & - & - & - \\
\hline & Mytilidae & $0,086(0,022)$ & - & $0,005(0,004)$ & - & - & - \\
\hline & Porphyra columbina & - & - & - & - & - & $9,38(2,97)$ \\
\hline & Rhodophyta & - & $0,35(0,18)$ & - & - & - & - \\
\hline
\end{tabular}


Tabla 5. Abundancia (ind $100 \mathrm{~cm}^{-2} \mathrm{dia}^{-1}$ ) promedio ( $\pm \mathrm{EE}$ ) de invertebrados y algas asentados por unidad de superficie y tiempo sobre la superficie de sustratos artificiales desplegados en la zona submareal de 6 sitios de estudio en el canal de Chacao / Mean ( \pm SE) abundance (ind $100 \mathrm{~cm}^{-2}$ day $^{-1}$ ) of settled marine invertebrates and algae settled per unit area and time on the surface of artificial substrates deployed in the rocky intertidal zone from 6 study sites at the Chacao channel

\begin{tabular}{lcccccc}
\hline Especie & Corona & Chocoi & Amazonas & Young & Caulin & Chilén \\
\hline Amphipoda & $0,575(0,18)$ & - & $0,183(0,049)$ & $0,008(0,008)$ & - & - \\
Anthozoa & - & - & $0,008(0,008)$ & $0,017(0,011)$ & - & - \\
Ascidiacea & - & - & - & $0,008(0,008)$ & - & - \\
Aulacomya ater & - & - & $0,008(0,008)$ & - & - \\
Bivalvia & $-108(0,057)$ & $0,040(0,040)$ & $0,008(0,008)$ & $0,025(0,013)$ & $0,493(0,120)$ & $0,009(0,009)$ \\
Bryozoa 1 & $0,192(0,075)$ & $0,066(0,024)$ & $0,075(0,040)$ & $0,050(0,028)$ & $0,083(0,031)$ & $0,009(0,009)$ \\
Bryozoa 2 & $0,267(0,108)$ & - & $0,117(0,083)$ & $0,008(0,008)$ & - & - \\
Bryozoa 3 & - & - & $0,042(0,019)$ & - & - & $0,009(0,009)$ \\
Porifera & $0,100(0,041)$ & $0,046(0,033)$ & $0,025(0,017)$ & - & - & - \\
Phaeophyta (esporas) & $0,408(0,182)$ & $2,533(0,849)$ & $0,967(0,751)$ & $0,050(0,050)$ & - & - \\
Rhodophyta (esporas) & $0,117(0,092)$ & - & $0,025(0,013)$ & - & - & - \\
Isopoda & $0,025(0,013)$ & - & - & - & - & - \\
Larvas cypris & $0,008(0,008)$ & - & - & $0,0125(0,012)$ & - & - \\
Mytilidae & $0,025(0,017)$ & $0,107(0,072)$ & $0,025(0,013)$ & $0,017(0,011)$ & $0,605(0,147)$ & $1,086(0,120)$ \\
Serpulidae & & & & & - \\
\hline
\end{tabular}

al 2010 para el desembarque de la ascidia P. chilensis y el cirrípedo gigante $A$. psittacus indica que el desembarque promedio anual $( \pm \mathrm{EE})$ en toneladas por caleta presente en el área de estudio ( $n=7$ caletas) es de 492,63 $( \pm 91,50)$ y $177,45( \pm 58,27)$ ton, respectivamente. En el caso de $P$. chilensis estos valores corresponderían a un 76,59 y $39,51 \%$ del desembarque anual promedio de la región y del país para el mismo periodo de análisis. En el caso de A. psittacus estos valores corresponderían a un 98,53 y $61,15 \%$ del desembarque anual promedio de la región y del país para el mismo periodo de análisis. Desde el punto de vista de la biomasa que puede alcanzar en el ambiente natural, destaca también la especie del mitílido comestible $M$. chilensis. Sin embargo, la información referente a $M$. chilensis proviene exclusivamente de producciones asociadas a 42 centros de cultivo que operan en la zona de estudio (consulta ciudadana online SIAC, No 460054512 SERNAPESCA). Para el mismo periodo de análisis utilizado para $P$. chilensis y A. psittacus, se registra un desembarque promedio $( \pm$ EE) de 3391,45 ( \pm $730,85)$ ton anuales asociadas a los centros de cultivo que operan en la zona de estudio (i.e., Región de los
Lagos).

\section{Discusión}

Este trabajo constituye el primer esfuerzo para recopilar la información sobre organismos marinos bio-incrustantes presentes en la costa de Chile. Además, para el caso particular de la zona del canal de Chacao, constituye el primer esfuerzo en que se realizan: (1) inventarios directos obtenidos en terreno sobre especies bio-incrustantes presentes en sustratos duros naturales (i.e., zonas rocosas intermareales); (2) registros directos obtenidos en terreno sobre especies bio-incrustantes que habitan sustratos costeros manufacturados por el hombre (i.e., pilotes y defensas costeras); (3) registros de especies de invertebrados y algas que se asentaron en sustratos artificiales experimentales (i.e., placas de acrílico cubiertas con antideslizante) desplegados en la zona inter y submareal. La revisión para la costa de Chile y las observaciones realizadas en sustratos artificiales intermareales del canal de Chacao indica que existen 211 y 32 especies de bio-incrustantes, respectivamente. Sin 
embargo, para el canal de Chacao, considerando las observaciones in situ, el estudio indica que existen además 133 especies de macro-algas e invertebrados sobre sustratos duros intermareales que potencialmente pueden constituirse en bio-incrustantes de estructuras artificiales. Esta información es de relevancia para proyectos futuros que pretendan instalar estructuras en esta zona y cuyo funcionamiento o integridad este afectada por su interacción con bio-incrustantes marinos (i.e., recuperadores de energía mareomotriz, pilotes de puentes, torres de transmisión eléctrica y defensas costeras). Para las costas de Chile se han registrado un total de 51 especies marinas introducidas (Castilla \& Neill 2009). Considerando que la zona norte de la isla de Chiloé alberga una importante actividad de acuicultura y que es considerado un lugar potencial para la introducción de especies (Camus 2005, Castilla \& Neill 2009), llama la atención el bajo número de especies introducidas $(n=6)$ que fueron registradas por este estudio en el canal de Chacao. Una posible explicación para este hecho es que los centros de cultivo se concentran en sectores más protegidos de la isla de Chiloé y no en el canal caracterizado por altas corrientes mareales (Hassan 2009). Sin embargo, el reducido tráfico de barcos con rutas internacionales no puede ser descartado como otra potencial explicación.

La estimación de la riqueza de especies a partir de la riqueza de especies inventariada es posible a través de métodos estadísticos (Palmer 1990, Chiarucci et al. 2003). El que no se alcanzaran valores asintóticos en los muestreos intermareales en 2 localidades en el borde insular del canal de Chacao sugieren que los muestreos no fueron suficientemente exhaustivos en dichas localidades o que la riqueza de especie era mayor. Sin embargo, el objetivo del presente estudio fue disponer de un inventario inicial de las especies que pudiesen constituirse en importantes bio-incrustantes sobre estructuras artificiales. En este sentido, es poco probable que especies con bajas abundancias y pequeño tamaño no estén representadas en los listados de especies de este estudio. Esto sugiere que gran parte de las especies que pudiesen constituir el EBIM en la zona del canal de Chacao han sido incorporadas. De particular importancia, por la biomasa que pueden alcanzar en el ambiente natural, destacan 3 especies de invertebrados marinos característicos de la zona de estudio en el canal de Chacao: el cirrípedo gigante $A$. psittacus, la ascidia $P$. chilensis y el mitílido M. chilensis. No se evaluó directamente la biomasa de estas especies en la zona de estudio en el canal de Chacao; sin embargo, el análisis de los desembarques de A. psittacus y $P$. chilensis y las producciones de $M$. chilensis asociadas a la zona de estudio sugieren que si estas especies se asientan y crecen con éxito sobre estructuras artificiales pueden constituirse en importantes EBIM. Las unidades de asentamiento artificial, desplegadas en la zona inter y submareal de la zona de estudio, no detectaron asentamiento de $P$. chilensis y A. psittacus. Sin embargo, es necesario considerar que los sustratos de asentamiento sólo fueron desplegados por un corto periodo de tiempo y no capturan la estacionalidad ni el uso de otros tipos de sustratos. Es bien conocido que el asentamiento de invertebrados marinos sobre sustratos artificiales y naturales requiere de la previa formación de una película bacteriana (Wieczorek \& Todd 1997) y en el caso de $P$. chilensis el asentamiento se registra en sustratos artificiales acondicionados con con-específicos adultos o principalmente sobre estos (Manríquez \& Castilla 2007). En consecuencia, es probable que el tiempo en que las unidades de asentamiento estuvieron expuestas al ambiente no fuera suficiente para que estas se acondicionaran adecuadamente para facilitar el asentamiento. El asentamiento de mitílidos se registra principalmente en sustratos filamentosos naturales (i.e., filamentos adhesivos o bisos de mitílidos previos) y artificiales (Martínez \& Navarrete 2002). En el presente estudio sustratos filamentosos no fueron utilizados; sin embargo, los mitílidos no identificados fueron registrados en las placas de asentamiento desplegadas en la zona submareal. Debido a que $M$. chilensis es una especie muy común en centros de cultivo y bancos naturales, es altamente probable que estos mitílidos asentados correspondan a dicha especie de mitílido. Además, es necesario considerar que el periodo durante el cual los sustratos de asentamiento experimental fueron expuestos en el ambiente natural pudiese no coincidir con la ventana temporal de reproducción/asentamiento de las 3 especies potenciales bio-incrustantes. Además, en el presente estudio las placas de asentamiento sólo fueron desplegadas en el fondo y zonas rocosas intermareales de los sitios de estudio en el canal de Chacao y no se evaluó el asentamiento en placas colocadas en la columna de agua a diferentes profundidades. El asentamiento de M. chilensis en los colectores utilizados en los centros de cultivo en Chiloé se registra desde la superficie hasta los $20 \mathrm{~m}$ de profundidad (PH Manríquez observ. pers.). Esto sugiere que esta especie puede asentarse masivamente en estructuras artificiales desplegadas en 
el mar y en profundidades similares a las que se registran en los bancos naturales o recolectores. En los centros de cultivo de $M$. chilensis en la localidad de Yaldad (extremo sur de la isla de Chiloé, $43^{\circ} \mathrm{S}$ ), ejemplares de $P$. chilensis y A. psittacus se registran desde la superficie de las boyas y la línea madre hasta profundidades cercanas a los $20 \mathrm{~m}$ a lo largo de la extensión de las líneas de mitílidos (PH Manríquez observ. pers.). En Carelmapu (Fig. 1) las observaciones indicaron desembarques masivos de estas 2 especies, lo que se coincide con el análisis de los desembarques registrados en la zona de estudio en el canal de Chacao.

Una importante conclusión del presente estudio es que sustratos artificiales presentes en la zona intermareal del canal de Chacao proporcionan hábitats adecuados para EBIM de especies que también habitan sustratos naturales presentes sobre zonas rocosas intermareales cercanas. Como este estudio se concentró sólo en la descripción del EBIM presente en la zona intermareal rocosa, no consideró estudios para evaluar qué bioincrustantes se registran sobre sustratos rocosos a mayores profundidades. Sin embargo, debido a las altas biomasas extraídas de la ascidia $P$. chilensis y del cirrípedo gigante $A$. psittacus, como de biomasa producida de $M$. chilensis, es altamente probable que estas especies se registren además en estructuras artificiales sumergidas en el mar de las localidades de estudio en el canal de Chacao.

Las estructuras artificiales proporcionan un apropiado hábitat para el asentamiento de una proporción alta de las especies de invertebrados y algas que se registran en hábitats naturales cercanos (Connell 2001, Perkol-Finkel $\&$ Benayahu 2005). Sin embargo, existen diferencias entre los EBIM que se registran entre diferentes tipos de hábitats artificiales y entre estos hábitats y los hábitats naturales (Connell 2001, Cole et al. 2005). Una potencial causa de las diferencias entre los ensambles se ha atribuido a las diferencias existentes en la edad de los sustratos (Connell \& Glasby 1999). Para el canal de Chacao, en 4 de las 6 localidades en las que se utilizaron curvas de saturación de especies para estimar la riqueza de especies se obtuvo tal saturación. Esto indica que el esfuerzo de muestreo de 30 min fue suficiente para medir la riqueza de especies en la mayoría de los sitios de estudio.

La agregación de los EBIM constituye un serio problema para la infraestructura asociada a la acuicultura como para las mismas especies cultivadas (Fitridge et al. 2012). Además, los EBIM incrementan la fricción de draga hidrodinámica que se opone al desplazamiento normal de embarcaciones en el mar (Schultz et al. 1999, 2011; Schultz \& Swain 2000). Esto implica la necesidad de destinar tiempo y fondos para el control del establecimiento de los EBIM como para el mantenimiento de todo tipo de estructuras manufacturadas artificiales desplegadas en el mar. El presente estudio aporta con una descripción de la composición potencial de especies que podrían constituir el EBIM en la zona del canal de Chacao. Sin embargo, la interacción de estas especies y otras especies de bio-incrustantes presentes en el canal de Chacao con futuras estructuras artificiales como equipos recuperadores de energía mareomotriz, puentes o defensas costeras, requerirá el reconocimiento que el asentamiento de muchas especies de invertebrados y algas puede estar modulada por la naturaleza, superficie, orientación, profundidad y edad de tales estructuras (Glasby \& Connell 2001, Perkol-Finkel et al. 2006, Walker et al. 2007, Rule \& Smith 2007, Pacheco et al. 2010).

Estudios futuros serán necesarios para establecer con claridad qué componentes del EBIM descritos en el presente estudio podrían interactuar con estructuras manufacturadas por el hombre y desplegadas en el canal de Chacao. Estos estudios deberán incluir: (1) el uso de diferentes tipos de sustratos artificiales, incluidos aquellos similares a los utilizados en la fabricación del equipo; (2) estacionalidad en la confección de los listados de especies y en las evaluaciones del asentamiento de los bio-incrustantes; (3) evaluar el asentamiento en función de la profundidad. Esto permitirá disponer información más precisa del EBIM en el canal de Chacao y de la variación estacional/espacial. Con esta información se podrá predecir el asentamiento de los componentes del EBIM sobre estructuras artificiales desplegadas en el canal de Chacao, así como programar su mitigación y/o remoción desde su superficie para evitar la interferencia en la instalación y funcionamiento óptimo de estas estructuras.

\section{Agradecimientos}

Este trabajo fue financiado por el Proyecto FONDEF D09i1052 de CONICYT Chile 'Evaluación del recurso energético asociado a corrientes mareales en el Canal de Chacao para la selección e implementación de dispositivos recuperadores de energía'. Este proyecto fue cofinanciado por aportes de HidroChile S.A. y DICTUC S.A. y dirigido por el Dr. Rodrigo Cienfuegos, Departamento de Ingeniería Hidráulica y Ambiental, Escuela de Ingeniería, Pontificia 
Universidad Católica de Chile. Los autores agradecen a R. Cienfuegos y a M. Guerra por su apoyo y coordinación en las actividades de terreno. Nuestros agradecimientos también para J. Beltrán y N. Rozbaczylo, Departamento de Ecología, Pontificia Universidad Católica de Chile, por su ayuda en la identificación de algas y poliquetos y al Dr. M. Lee por la corrección de los textos en inglés. Durante el desarrollo de este proyecto PHM era parte del Proyecto ANILLOS ACT-132 y del Proyecto FONDECYT 1130839. Agradecemos los comentarios de 3 evaluadores anónimos que mejoraron la versión inicial del manuscrito.

\section{LITERATURA CITADA}

Aiken CM. 2008. Barotropic tides of the Chilean Inland Sea and their sensitivity to basin geometry. Journal of Geophysical Research 113, C08024 <doi:10.1029/ 2007JC004593>

Ambler RT \& JI Cañete. 1991. Asentamiento y reclutamiento de Pyura chilensis Molina 1782 (Urochordata: Ascidiacea) sobre placas artificiales suspendidas en la Bahía la Herradura, Coquimbo, Chile. Revista de Biología Marina 26(2): 403-413.

Astudillo JC, M Bravo, CP Dumont \& M Thiel. 2009. Detached aquaculture buoys in the SE Pacific: Potential dispersal vehicles for associated organisms. Aquatic Biology 5: 219-231.

Basilio CD, JI Cañete \& N Rozbaczylo. 1995. Polydora sp. (Spionidae) un poliqueto perforador de las valvas del ostión Argopecten purpuratus (Bivalvia: Pectinidae) en Bahía Tongoy, Chile. Revista de Biología Marina 30: 71-77.

Brattström H \& A Johanssen. 1983. Ecological and regional zoogeography of the marine benthic fauna of Chile. Sarsia 68: 289-339.

Bulleri F \& L Airoldi. 2005. Artificial marine structures facilitate the spread of nonindigenous green alga, Codium fragile spp.tomentosoides, in the north Adriatic Sea. Journal of Applied Ecology 42: 1063-1072.

Bulleri F \& MG Chapman. 2010. The introduction of coastal infrastructure as a driver of change in marine environments. Journal of Applied Ecology 47(1): 26-35.

Cáceres M, A Valle-Levinson \& L Atkinson. 2003. Observations of cross-channel structure of flow in an energetic tidal channel. Journal of Geophysical Research 108 C4, 3114 <doi: 10.1029/2001JC000968>

Camus PA. 2005. Introducción de especies en ambientes marinos Chilenos: no solo exóticas, no siempre evidentes. Revista Chilena de Historia Natural 78: 155-159.

Cañete JI \& RP Ambler. 1990. Growth and age determination in the spirorbid polychaete Romanchella pustulata KnightJones, 1978. Revista de Biología Marina 25(2): 147-164.
Cao W, T Yan, Z Li, J Li \& Z Cheng. 2013. Fouling acorn barnacles in China-a review. Chinese Journal of Oceanology and Limnology 31(4): 699-711.

Carranza A, O Defeo, M Beck \& JC Castilla. 2009. Linking fishing management and conservation in bioengineering species: the case of South American mussels (Mytilidae). Reviews in Fish Biology and Fisheries 19: 349-366.

Castilla JC. 1981. Perspectivas de investigación en estructura y dinámica de comunidades intermareales rocosas de Chile Central. II. Depredadores de alto nivel trófico. Medio Ambiente 5(1-2): 190-215.

Castilla JC. 2008. Una guía para la observación del litoral, 120 pp. Impresora Valus, Santiago.

Castilla JC \& PE Neill. 2009. Marine bioinvasions in the southeastern Pacific: status, ecology, economic impacts, conservation and management. In: Rilov G \& JA Crooks (eds). Biological invasions in marine ecosystems, pp. 439457. Springer, Berlin.

Castilla JC, NA Lagos \& M Cerda. 2004. Marine ecosystem engineering by the alien ascidian Pyura praeputialis on a mid-intertidal rocky shore. Marine Ecology Progress Series 268: 119-130.

Castilla JC, M Uribe, N Bahamonde, M Clarke, R Desqueyroux-Faúndez, I Kong, H Moyano, N Rozbaczylo, B Santelices, C Valdovinos \& P Zavala. 2005. Down under the Southeastern Pacific: marine non-indigenous species in Chile. Biological Invasions 7: 213-232.

Chiarucci A, NJ Enright, GL Perry, BP Miller \& BB Lamont. 2003. Performance of nonparametric species richness estimators in a high diversity plant community. Diversity and Distributions 9: 283-295.

Clarke M \& JC Castilla. 2000. Dos nuevos registros de ascidias (Tunicata: Ascidiacea) para la costa continental de Chile. Revista Chilena de Historia Natural 73(3): 503-510.

Cole VJ, TM Glasby \& MG Holloway. 2005. Extending the generality of ecological models to artificial floating habitats. Marine Environmental Research 60: 195-210.

Connell SD. 2001. Urban structures as marine habitats: an experimental comparison of the composition and abundance of subtidal epibiota among pilings, pontoons and rocky reefs. Marine Environmental Research 52(2): 115-125.

Connell SD \& TM Glasby. 1999. Do urban structures influence local abundance and diversity of subtidal epibiota? A case study from Sydney Harbour, Australia. Marine Environmental Research 47(4): 373-387.

Darwin C. 1854. A monograph on the Sub-class Cirripedia, with figures of all the species. The Ray Society. London 1851-1854.

Davis AR. 1995. Over-exploitation of Pyura chilensis (Ascidiacea) in southern Chile: the urgent need to establish marine reserves. Revista Chilena de Historia Natural 68: 107-116. 
Falcão A. 2010. Wave energy utilization: A review of the technologies. Renewable and Sustainable Energy Reviews 14(3): 899-918.

Fitridge I, T Dempster, J Guenther \& R de Nys. 2012. The impact and control of biofouling in marine aquaculture: a review. Biofouling 28(7): 664-669.

Glasby TM \& SD Connell. 1999. Urban structures as marine habitats. Ambio 28: 595-598.

Glasby TM \& SD Connell. 2001. Orientation and position of substrata have large effects on epibiotic assemblages. Marine Ecology Progress Series 214: 127-135.

Hassan G. 2009. Preliminary site selection - Chilean Marine Energy Resources. Report for the Inter-American Development Bank. Document 100513/BR/02, Issue 2: 169. <http://antiguo.minenergia.cl/minwww/export/sites/ default/05_Public_Estudios/descargas/estudios/ texto10.pdf>

Hillman RE. 1977. Techniques for monitoring reproduction and growth of fouling organisms at power plant intakes. In: Jensen LD (ed). Biofouling control procedures, p. 5, Mercel Dekkar, New York.

Jones C, J Lawton \& M Shachak. 1994. Organisms as ecosystem engineers. Oikos 69: 373-386.

Knott NA, AJ Underwood, MG Chapman \& TM Glasby. 2004. Epibiota on vertical and on horizontal surfaces on natural reefs and on artificial structures. Journal of the Marine Biological Association of the United Kingdom 84: 1117-1130.

Lancellotti DA \& JA Vásquez. 2000. Zoogeografía de macroinvertebrados bentónicos de la costa de Chile: Contribución para la conservación marina. Revista Chilena de Historia Natural 73: 99-129.

Langhamer O, D Wilhelmsson \& J Engström. 2009. Artificial reef effect and fouling impacts on offshore wave power foundations and buoys -a pilot study. Estuarine, Coastal and Shelf Science 82: 426-432.

Langhamer O, K Haikonen \& J Sundberg. 2010. Wave power -Sustainable energy or environmentally costly? A review with special emphasis on linear wave energy converters. Renewable and Sustainable Energy Reviews 14: 1329-1335.

López DA. 1994. Efecto de la estructura corporal de cirripedios en el resultado de interacciones bióticas. Tesis Doctoral, Pontificia Universidad Católica de Chile, Santiago, 181 pp.

López DA, BA López, C Burgos, SE Arriagada \& ML González. 2007. Consequences of base modification in hummocks of the barnacle Austromegabalanus psittacus. New Zealand Marine and Freshwater Research 41(3): 291-298.

López DA, BA López, CK Pham \& EJ Isidro. 2012. Potency of barnacle in aquaculture industry. In: Muchlisin Z (ed). Aquaculture, Chapter 15: 295-316. <http://www.intechopen.com/books/ aquaculture/potency-of-barnacle-in-aquaculture-industry>
Maar M, K Bolding, JK Petersen, JLS Hansen \& K Timmermann. 2009. Local effects of blue mussels around turbine foundations in an ecosystem model of Nysted offshore wind farm, Denmark. Journal of Sea Research 62: 159-174.

Manríquez PH \& JC Castilla. 2007. Roles of larval behaviour and microhabitat traits in determining spatial aggregations in the ascidian Pyura chilensis. Marine Ecology Progress Series 305: 113-125.

Martínez P \& SA Navarrete. 2002. Temporal and spatial variation in settlement of the gastropod Concholepas concholepas in natural and artificial substrata. Journal of the Marine Biological Association of the United Kingdom 82: $257-264$

Navarrete SA, B Broitman, EA Wieters, GR Finke, RM Venegas \& A Sotomayor. 2002. Recruitment of intertidal invertebrates in the southeast Pacific: Interannual variability and the 1997-1998 El Niño. Limnology and Oceanography 47(3): 791-802.

Neill P, O Alcalde, S Faugeron, SA Navarrete \& JA Correa. 2006. Invasion of Codium fragile ssp. Tomentosoides in northern Chile: a new threat for Gracilaria farming. Aquaculture 259: 202-221.

Pacheco A \& A Garate. 2005. Bioincrustantes en estructuras de cultivo de Argopecten purpuratus en Bahía Samanco, Perú. Ecología Aplicada 4(1,2): 149-152.

Pacheco AS, J Laudien, M Thiel, O Heilmayer \& M Oliva. 2010. Hard-bottom succession of subtidal epibenthic communities colonizing hidden and exposed surfaces off northern Chile. Scientia Marina 74: 147-154

Palmer MW. 1990. The estimation of species richness by extrapolation. Ecology 71: 1195-1198.

Perkol-Finkel S \& Y Benayahu. 2005. Recruitment of benthic organisms onto a planned artificial reef: shifts in community structure one decade post-deployment. Marine Environmental Research 59: 79-99.

Perkol-Finkel S, G Zilman, I Sella, T Miloth \& Y Benayahu. 2006. Floating and fixed artificial reefs: the effect of substratum motion on benthic communities. Marine Ecology Progress Series 317: 9-20.

Prado L \& JC Castilla. 2006. The bioengineer Perumytilus purpuratus (Mollusca: Bivalvia) in central Chile: biodiversity, structural complexity and heterogeneity. Journal of the Marine Biological Association of the United Kingdom 86: 417-421.

Railkin AI. 2004. Marine biofouling. Colonization processes and defenses, 304 pp. CRC Press, Boca Raton.

Rajagopal S, J Azariah \& KVK Nair. 1990. Ecology of fouling organisms in Edaiyur backwater, Kalpakkan. Mahasagar 23(1): 29-41. 
Rebolledo ME. 1998. El erizo negro Tetrapygus niger (Molina, 1782) como organismo controlador de biofouling asociado al cultivo suspendido de Argopecten purpuratus (Lamarck, 1819) en Bahía Tongoy, IV Región. Tesis de Biólogo Marino, Facultad de Ciencias del Mar, Universidad Católica del Norte, Coquimbo, 110 pp.

Richmond MD \& R Seed. 1991. A review of marine macrofouling communities with a special reference to animal fouling. Biofouling 3: 151-168.

Rule MJ \& SD Smith. 2007. A Depth-associated patterns in the development of benthic assemblages on artificial deployed on shallow, subtropical reefs. Journal of Experimental Marine Biology and Ecology 345: 38-51.

Schultz MP \& GW Swain. 2000. The influence of biofilms on skin friction drag. Biofouling 15(1-3): 129-139.

Schultz MP, CJ Kavanagh \& G Swain. 1999. Hydrodynamic forces on barnacles: implications on detachment from fouling-release surfaces. Biofouling 13(4): 323-335.

Schultz MP, JA Bendick, ER Holm \& WM Hertel. 2011. Economic impact of biofouling on a naval surface ship. Biofouling 27(1): 87-98.

Sepúlveda R, JM Cancino \& M Thiel. 2003. The peracarid epifauna associated with the ascidian Pyura chilensis (Molina, 1782) (Ascidiacea: Pyuridae). Journal of Natural History 37(13): 1555-1569.

SERNAPESCA. 2010. Anuario estadístico de pesca. Servicio Nacional de Pesca, Ministerio de Economía, Fomento y Turismo, Valparaíso. <www.sernapesca.cl>

Shaw S, MJ Cremers \& G Palmers. 2002. Enabling offshore wind developments. European Wind Energy Association, Brussels <http://www2.ewea.org/documents/offshore\%20\%20EWEA\%20version\%20.pdf $>$

Smith SDA \& MJ Rule. 2002. Artificial substrata in a shallow sublittoral habitat: do they adequately represent natural habitats of the local species pool? Journal of Experimental Marine Biology and Ecology 277: 25-41.

Soberón J \& J Llorente. 1993. The use of species accumulation functions for the prediction of species richness. Conservation Biology 7: 480-488.
Svane I \& JK Petersen. 2001. On the problems of epibioses, fouling and artificial reefs, a review. Marine Ecology 22: 169-188.

Thompson GG, PC Withers, ER Pianka \& SA Thompson. 2003. Assessing biodiversity with species accumulation curves; inventories of small reptiles by pit-trapping in Western Australia. Austral Ecology 28: 361-383.

Ugland KI, JS Gray \& KE Ellingsen. 2003. The speciesaccumulation curve and estimation of species richness. Journal of Animal Ecology 72: 888-897.

Uribe E, C Lodeiros, E Felix-Pico \& I Etchepare. 2001. Epibiontes en pectínidos de iberoamérica. En: MaedaMartínez AN (ed). Los moluscos Pectínidos de Iberoamérica. Ciencia y Acuicultura 13: 249-266. Limusa, México.

Valdivia N, A Heidemann, M Thiel, M Molis \& M Whal. 2005. Effects of disturbance on the diversity of hard-bottom macrobenthic communities on the coast of Chile. Marine Ecology Progress Series 299: 45-54.

Vásquez JA. 1983. Pyura chilensis Molina, 1782 en el Norte del Perú (Ascidiacea, Pyuridae). Boletín de la Sociedad de Biología de Concepción 54: 171-172.

Viviani CA \& LH DiSalvo. 1980. Biofouling in a north-central Chilean coastal bay. In: Proceedings of V Congreso Internacional de Corrosión Marina e Incrustaciones, Barcelona, pp. 69-74.

Von Plessing MT. 1981. Desarrollo de la comunidad adherente en los cultivos de mitílidos. Yaldad, Chiloé, Chile. Tesis de Licenciado en Biología, Facultad de Ciencias Biológicas y de Recursos Naturales, Universidad de Concepción, Concepción, 82 pp.

Walker SJ, TA Schlacher \& MA Schlacher-Hoenlinger. 2007. Spatial heterogeneity of epibenthos on artificial reefs: fouling communities in the early stages of colonization on an East Australian shipwreck. Marine Ecology 28: 345-445.

Wieczorek SK \& CD Todd. 1997. Inhibition and facilitation of bryozoan and ascidian settlement by natural multi-species biofilms: effects of film age and roles of active and passive larval attachment. Marine Biology 128: 468-473.

Yan T \& WX Yan. 2003. Fouling of offshore structures in China -a review. Biofouling 19: 133-138. 
Anexo 1. Registro fotográfico de las placas de asentamiento instaladas sobre sustratos duros naturales en zonas rocosas intermareales (a) y sustratos duros artificiales (b). El inserto superior en (a) corresponde a una placa de acrílico transparente cubierta con cinta antideslizante (3M \%). En ambas fotografías la línea blanca corresponde a 0,5 m / Photographic records of the settlement plates placed in natural hard bottom intertidal (a) and artificial (b) substrates. In the top insert in (a) correspond to a Plexiglas plate cover with slip-resistant tape $\left(3 \mathrm{M}^{\circ}\right)$. In both pictures the white line depicts $0.5 \mathrm{~m}$
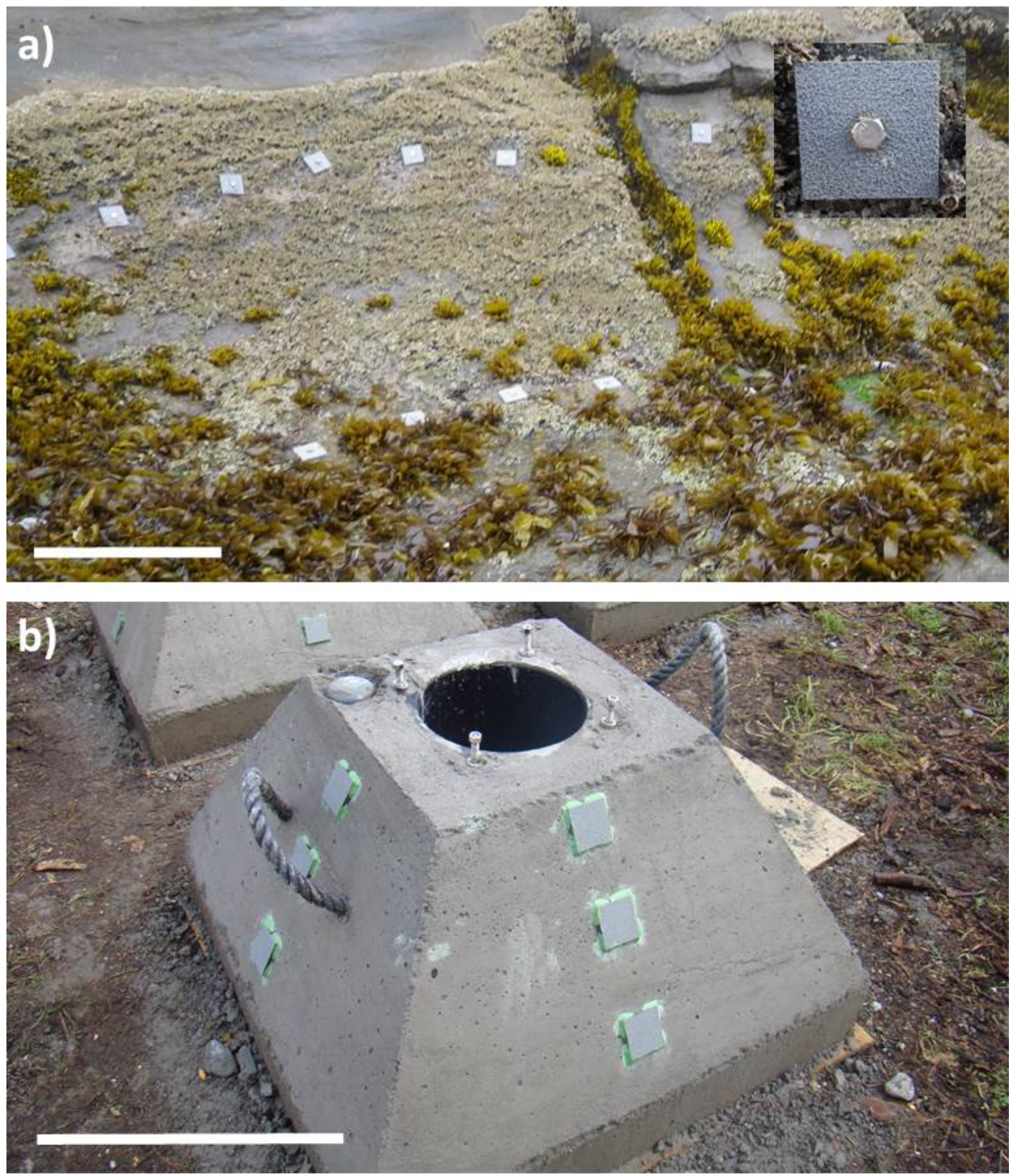
Anexo 2. Especies de bio-incrustantes $(n=211)$ registradas en Chile según la literatura existente. Se incluye información sobre el grupo taxonómico, nombre científico y zona donde se realizó el registro. Además, se incluye información sobre si corresponde a una especie móvil (M), sésil (S), nativa (N), introducida (I) y la referencia correspondiente. En la última columna se indican si las especies fueron registradas en la zona del canal de Chacao en el presente estudio $(n=7)$ / Bio-incrustant species $(n=211)$ recorded in Chile according the literature. Information regarding the taxonomic group, scientific name and the zone were the record was made is included. Moreover, information regarding if the species is mobile (M), sessile (S), native (N), introduced (I) and the corresponding reference is also included. In the last column if the species was recorded in the Chacao channel by the present study $(n=7)$ is also included

\begin{tabular}{|c|c|c|c|c|c|}
\hline Phylum / División & Nombre científico & Zona & $\mathrm{M} / \mathrm{S}$ & $\mathrm{N} / \mathrm{I}$ & Referencia \\
\hline \multirow{5}{*}{ Chlorophyta } & Bryopsis rhizophora & Norte & $\mathrm{S}$ & $\mathrm{N}$ & Astudillo et al. 2009 \\
\hline & Cladophora spp. & Norte & $\mathrm{S}$ & - & Uribe et al. 2001 \\
\hline & Codium fragile & Norte & $\mathrm{S}$ & I & $\begin{array}{l}\text { Uribe et al. 2001, Castilla et al. 2005, Neill et al. 2006, } \\
\text { Astudillo et al. 2009, Castilla \& Neill } 2009\end{array}$ \\
\hline & Enteromorpha sp. & Norte & $\mathrm{S}$ & - & Viviani \& DiSalvo 1980, Uribe et al. 2001 \\
\hline & Ulva sp. & Norte/Sur & $\mathrm{S}$ & - & $\begin{array}{l}\text { Von Plessing 1981, Rebolledo 1998, Uribe et al. 2001, } \\
\text { Valdivia et al. 2005, Astudillo et al. } 2009\end{array}$ \\
\hline \multirow[t]{14}{*}{ Phaeophyta } & Amphipleura rutilans & Sur & $\mathrm{S}$ & $\mathrm{N}$ & Von Plessing 1981 \\
\hline & Biddulphia sp. & Sur & $\mathrm{S}$ & - & Von Plessing 1981 \\
\hline & Colpomenia sp. & Norte & $\mathrm{S}$ & - & Astudillo et al. 2009 \\
\hline & Diatomeas & Norte & $\mathrm{S}$ & - & Viviani \& DiSalvo 1980 \\
\hline & Ectocarpaceae & Norte & $\mathrm{S}$ & - & Valdivia et al. 2005 \\
\hline & Ectocarpus acutus & Norte & $\mathrm{S}$ & $\mathrm{N}$ & Astudillo et al. 2009 \\
\hline & Ectocarpus sp. & Sur & $\mathrm{S}$ & - & Von Plessing 1981 \\
\hline & Endarachne binghamiae & Norte & $\mathrm{S}$ & $\mathrm{N}$ & Uribe et al. 2001 \\
\hline & Hincksia granulosa & Norte & $\mathrm{S}$ & $\mathrm{N}$ & Uribe et al. 2001, Astudillo et al. 2009 \\
\hline & Macrocystis pyrifera & Sur & $\mathrm{S}$ & $\mathrm{N}$ & Von Plessing 1981 \\
\hline & Petalonia fascia & Norte/Sur & $\mathrm{S}$ & $\mathrm{N}$ & Von Plessing 1981, Astudillo et al. 2009 \\
\hline & Phaeophyceae & Norte & $\mathrm{S}$ & - & Valdivia et al. 2005 \\
\hline & Ralfsiaceae & Norte & $\mathrm{S}$ & - & Valdivia et al. 2005 \\
\hline & Scytosiphon lomentaria & Norte/Sur & $\mathrm{S}$ & $\mathrm{N}$ & Von Plessing 1981, Uribe et al. 2001, Astudillo et al. 2009 \\
\hline \multirow[t]{13}{*}{ Rhodophyta } & Antithamnion densum & Norte & $\mathrm{S}$ & $\mathrm{N}$ & Valdivia et al. 2005, Astudillo et al. 2009 \\
\hline & Corallina officinalis & Norte & $\mathrm{S}$ & $\mathrm{N}$ & Astudillo et al. 2009 \\
\hline & Cryptonemia obovata & Norte & $\mathrm{S}$ & $\mathrm{N}$ & Uribe et al. 2001 \\
\hline & Ectocarpus sp. & Norte & $\mathrm{S}$ & - & Viviani \& DiSalvo 1980 \\
\hline & Gelidium spp. & Norte & $\mathrm{S}$ & - & Astudillo et al. 2009 \\
\hline & Heterosiphonia sp. & Norte & $\mathrm{S}$ & - & Viviani \& DiSalvo 1980 \\
\hline & Lithothamnioides & Norte & $\mathrm{S}$ & - & Viviani \& DiSalvo 1980 \\
\hline & Polysiphonia mollis & Norte & $\mathrm{S}$ & $\mathrm{N}$ & Valdivia et al. 2005, Astudillo et al. 2009 \\
\hline & Polysiphonia spp. & Norte & $\mathrm{S}$ & - & Uribe et al. 2001, Valdivia et al. 2005 \\
\hline & Porphyropsis coccinea & Norte & $\mathrm{S}$ & $\mathrm{N}$ & Uribe et al. 2001 \\
\hline & Rhodymenia corallina & Norte & $\mathrm{S}$ & $\mathrm{N}$ & Uribe et al. 2001 \\
\hline & Rhodymenia sp. & Norte & $\mathrm{S}$ & - & Astudillo et al. 2009 \\
\hline & Stenogramme interrupta & Norte & $\mathrm{S}$ & $\mathrm{N}$ & Uribe et al. 2001 \\
\hline \multirow[t]{4}{*}{ Porifera } & Cliona chilensis & Norte & $\mathrm{S}$ & $\mathrm{N}$ & Uribe et al. 2001 \\
\hline & Leocosolenia variabilis & Norte & $\mathrm{S}$ & $\mathrm{N}$ & Viviani \& DiSalvo 1980, Valdivia et al. 2005 \\
\hline & Porifera 1 & Norte & $\mathrm{S}$ & - & Astudillo et al. 2009 \\
\hline & Porifera 2 & Norte & $\mathrm{S}$ & - & Astudillo et al. 2009 \\
\hline \multirow[t]{17}{*}{ Cnidaria } & Alcyonarians sp. & Sur & $\mathrm{S}$ & - & Viviani \& DiSalvo 1980 \\
\hline & Anthothoe chilensis & Norte & $\mathrm{S}$ & $\mathrm{N}$ & Astudillo et al. 2009 \\
\hline & Campanularia sp. & Norte & $\mathrm{S}$ & - & Viviani \& DiSalvo 1980, Uribe et al. 2001 \\
\hline & Cordylophora sp. & Sur & $\mathrm{S}$ & - & Viviani \& DiSalvo 1980 \\
\hline & Eudembrium sp. & Norte & $\mathrm{S}$ & - & Uribe et al. 2001 \\
\hline & Hidractinia sp. & Norte & $\mathrm{S}$ & - & Uribe et al. 2001 \\
\hline & Hydrozoa 1 & Norte & $\mathrm{S}$ & - & Astudillo et al. 2009 \\
\hline & Hydrozoa 2 & Norte & $\mathrm{S}$ & - & Astudillo et al. 2009 \\
\hline & Hydrozoa 3 & Norte & $\mathrm{S}$ & - & Astudillo et al. 2009 \\
\hline & Hydrozoa 4 & Norte & $\mathrm{S}$ & - & Astudillo et al. 2009 \\
\hline & Hydrozoa 5 & Norte & $\mathrm{S}$ & - & Astudillo et al. 2009 \\
\hline & Hydrozoa 6 & Norte & $\mathrm{S}$ & - & Astudillo et al. 2009 \\
\hline & Obelia dichotoma & Sur & $\mathrm{S}$ & $\mathrm{N}$ & Von Plessing 1981 \\
\hline & Obelia sp. & Norte & $\mathrm{S}$ & - & $\begin{array}{l}\text { Viviani \& DiSalvo 1980, Uribe et al. 2001, Valdivia et al. } \\
\text { 2005, Astudillo et al. } 2009\end{array}$ \\
\hline & Phymactis clematis & Norte & S & $\mathrm{N}$ & $\begin{array}{l}\text { Valdivia et al. 2005, Astudillo et al. 2009, presente } \\
\text { estudio. }\end{array}$ \\
\hline & Plumularia setacea & Norte & $\mathrm{S}$ & $\mathrm{N}$ & Valdivia et al. 2005, Astudillo et al. 2009 \\
\hline & Tubularia sp. & Norte & $\mathrm{S}$ & - & $\begin{array}{l}\text { Viviani \& DiSalvo 1980, Uribe et al. 2001, Valdivia et al. } \\
2005\end{array}$ \\
\hline
\end{tabular}


Anexo 2. Continuación / continued

\begin{tabular}{|c|c|c|c|c|c|}
\hline \multirow[t]{5}{*}{ Platyhelminthes } & Turbellaria 1 & Norte & M & - & Astudillo et al. 2009 \\
\hline & Turbellaria 2 & Norte & M & - & Astudillo et al. 2009 \\
\hline & Turbellaria 3 & Norte & M & - & Astudillo et al. 2009 \\
\hline & Turbellaria 4 & Norte & M & - & Astudillo et al. 2009 \\
\hline & Turbellaria 5 & Norte & M & - & Astudillo et al. 2009 \\
\hline \multirow[t]{3}{*}{ Nemertea } & Nemertea 1 & Norte & M & - & Astudillo et al. 2009 \\
\hline & Nemertea 2 & Norte & M & - & Astudillo et al. 2009 \\
\hline & Nemertea 3 & Norte & M & - & Astudillo et al. 2009 \\
\hline \multirow[t]{11}{*}{ Ectoprocta } & Alcyonidium sp. & Norte & $\mathrm{S}$ & - & Valdivia et al. 2005 \\
\hline & Bryozoa 1 & Norte & $\mathrm{S}$ & - & Astudillo et al. 2009 \\
\hline & Bryozoa 2 & Norte & $\mathrm{S}$ & - & Astudillo et al. 2009 \\
\hline & Bryozoa 3 & Norte & $\mathrm{S}$ & - & Astudillo et al. 2009 \\
\hline & Bugula flabellata & Norte & $\mathrm{S}$ & I & $\begin{array}{l}\text { Uribe et al. 2001, Castilla et al. 2005, Valdivia et al. 2005, } \\
\text { Astudillo et al. } 2009 \text {, Castilla \& Neill } 2009\end{array}$ \\
\hline & Bugula neritina & Norte & $\mathrm{S}$ & I & $\begin{array}{l}\text { Rebolledo 1998, Uribe et al. 2001, Castilla et al. 2005, } \\
\text { Valdivia et al. 2005, Astudillo et al. 2009, Castilla \& Neill } \\
2009\end{array}$ \\
\hline & Conopeum sp. & Sur & $\mathrm{S}$ & - & Viviani \& DiSalvo 1980 \\
\hline & Corophium sp. & Norte & $\mathrm{S}$ & - & Viviani \& DiSalvo 1980 \\
\hline & Cryptosula pallasiana & Norte & $\mathrm{S}$ & $\mathrm{N}$ & Astudillo et al. 2009 \\
\hline & Membraniphora hyadesi & Sur & $\mathrm{S}$ & $\mathrm{N}$ & Von Plessing 1985 \\
\hline & Membraniphora isabelleana & Norte & $\mathrm{S}$ & $\mathrm{N}$ & Valdivia et al. 2005, Astudillo et al. 2009 \\
\hline \multirow[t]{4}{*}{ Mollusca: Bivalvia } & Argopecten purpuratus & Norte & M & $\mathrm{N}$ & Uribe et al. 2001, Astudillo et al. 2009 \\
\hline & Brachidontes granulata & Norte & $\mathrm{S}$ & $\mathrm{N}$ & Astudillo et al. 2009 \\
\hline & Mytilus chilensis & Sur & $\mathrm{S}$ & $\mathrm{N}$ & Von Plessing 1981, presente estudio. \\
\hline & Semimytilus algosus & Norte/Sur & $\mathrm{S}$ & $\mathrm{N}$ & $\begin{array}{l}\text { Viviani \& DiSalvo 1980, Rebolledo 1998, Uribe et al. } \\
\text { 2001, Valdivia et al. 2005, Astudillo et al. } 2009\end{array}$ \\
\hline \multirow[t]{30}{*}{ Mollusca: Gastropoda } & Concholepas concholepas & Norte & M & $\mathrm{N}$ & Uribe et al. 2001, Astudillo et al. 2009, presente estudio. \\
\hline & Crassilabrum crassilabrum & Norte & M & $\mathrm{N}$ & Uribe et al. 2001 \\
\hline & Crepidula fecunda & Sur & M & $\mathrm{N}$ & Von Plessing 1981 \\
\hline & Crepidula sp. & Norte & M & - & Valdivia et al. 2005, Astudillo et al. 2009 \\
\hline & Crepipatella sp. & Norte & M & - & Uribe et al. 2001 \\
\hline & Crepipatella dilatata & Norte & M & $\mathrm{N}$ & Viviani \& DiSalvo 1980 \\
\hline & Crucibulum quiriquinae & Norte & M & $\mathrm{N}$ & Uribe et al. 2001 \\
\hline & Crucibulum sp. & Norte & M & - & Astudillo et al. 2009 \\
\hline & Doto uva & Norte & M & $\mathrm{N}$ & Astudillo et al. 2009 \\
\hline & Fissurella cumingi & Norte & M & $\mathrm{N}$ & Astudillo et al. 2009 \\
\hline & Fissurella latimarginata & Norte & M & $\mathrm{N}$ & Astudillo et al. 2009 \\
\hline & Fissurella spp. & Norte/Sur & M & - & Von Plessing 1981, Uribe et al. 2001, Astudillo et al. 2009 \\
\hline & Hancockia schoeferti & Norte & M & $\mathrm{N}$ & Astudillo et al. 2009 \\
\hline & Hiatella solida & Norte & M & $\mathrm{N}$ & Uribe et al. 2001 \\
\hline & Hormomya granulata & Norte & M & $\mathrm{N}$ & Viviani \& DiSalvo 1980 \\
\hline & Mitrella unifasciata & Norte & M & $\mathrm{N}$ & Uribe et al. 2001 \\
\hline & Mitrella sp. & Norte & M & - & Astudillo et al. 2009 \\
\hline & Nassaridae & Norte & M & - & Astudillo et al. 2009 \\
\hline & Nassarius sp. & Norte & M & - & Uribe et al. 2001, Astudillo et al. 2009 \\
\hline & Nudibranchia indeterminado & Norte & M & - & Astudillo et al. 2009 \\
\hline & Phidiana sp. & Norte & $\mathrm{M}$ & - & Rebolledo 1998, Uribe et al. 2001 \\
\hline & Phidiana lottini & Norte & M & $\mathrm{N}$ & Astudillo et al. 2009 \\
\hline & Platydoris sp. & Norte & M & - & Astudillo et al. 2009 \\
\hline & Prisogaster sp. & Norte & M & - & Astudillo et al. 2009 \\
\hline & Scurria (Lottia) orbignyi & Norte & M & $\mathrm{N}$ & Astudillo et al. 2009 \\
\hline & Scurria viridula & Norte & M & $\mathrm{N}$ & Astudillo et al. 2009 \\
\hline & Siphonaria lessoni & Norte & M & $\mathrm{N}$ & Astudillo et al. 2009 , presente estudio. \\
\hline & Tegula sp. & Norte & M & - & Uribe et al. 2001 \\
\hline & Thecacera darwini & Norte & M & $\mathrm{N}$ & Astudillo et al. 2009 \\
\hline & Trochita calyptraeformis & Norte & M & $\mathrm{N}$ & Viviani \& DiSalvo 1980, Uribe et al. 2001 \\
\hline
\end{tabular}


Anexo 2. Continuación / continued

\begin{tabular}{|c|c|c|c|c|c|}
\hline Mollusca: Polyplacophora & $\begin{array}{l}\text { Chiton granosus } \\
\text { Chiton } \mathrm{sp} . \\
\text { Tonicia } \mathrm{sp} .\end{array}$ & $\begin{array}{l}\text { Sur } \\
\text { Norte } \\
\text { Norte }\end{array}$ & $\begin{array}{l}\mathrm{M} \\
\mathrm{M} \\
\mathrm{M}\end{array}$ & $\begin{array}{l}N \\
- \\
-\end{array}$ & $\begin{array}{l}\text { Von Plessing 1981, presente estudio. } \\
\text { Astudillo et al. } 2009 \\
\text { Astudillo et al. } 2009\end{array}$ \\
\hline Annelida & $\begin{array}{l}\text { Amaeana sp. } \\
\text { Autolytus simplex } \\
\text { Autolytus sp. } \\
\text { Branchiomma sp. } \\
\text { Capitella capitata } \\
\text { Cirratulus } \text { sp. } \\
\text { Cirratulidae } 1 \\
\text { Cirratulidae } 2 \\
\text { Dodecaceria opulens } \\
\text { Eulalia } \text { sp. } \\
\text { Halosydna patagonica } \\
\\
\text { Harmothoe sp. } \\
\text { Lumbrinereis sp. } \\
\text { Naineris chilensis } \\
\text { Nereis grubei } \\
\text { Nicolea chilensis } \\
\text { Orbiniidae } \\
\text { Odontosyllis sp. } \\
\text { Paleanotus } \text { sp. } \\
\text { Platynereis australis } \\
\text { Platynereis australis } \\
\text { megalhaensis } \\
\text { Polycirrus sp. } \\
\text { Polydora sociales } \\
\text { Polydora sp. } \\
\text { Polynoidae } \\
\text { Pseudonereis gallapagensis } \\
\text { Romanchella postulata } \\
\text { Sabella pusilla } \\
\text { Steggoa magalhaensis } \\
\text { Syllidae } \\
\text { Typosyllis magdalena }\end{array}$ & $\begin{array}{l}\text { Norte } \\
\text { Norte } \\
\text { Norte } \\
\text { Norte } \\
\text { Norte } \\
\text { Norte } \\
\text { Norte } \\
\text { Norte } \\
\text { Norte } \\
\text { Norte } \\
\text { Norte/Sur }\end{array}$ & $\begin{array}{l}\text { M } \\
M \\
M \\
S \\
M \\
S \\
S \\
S \\
S \\
M \\
M\end{array}$ & $\begin{array}{c}- \\
\mathrm{N} \\
- \\
- \\
\mathrm{N} \\
- \\
- \\
- \\
\mathrm{N} \\
- \\
\mathrm{N}\end{array}$ & $\begin{array}{l}\text { Astudillo et al. } 2009 \\
\text { Astudillo et al. } 2009 \\
\text { Astudillo et al. } 2009 \\
\text { Astudillo et al. } 2009 \\
\text { Uribe et al. } 2001 \\
\text { Astudillo et al. } 2009 \\
\text { Astudillo et al. } 2009 \\
\text { Astudillo et al. } 2009 \\
\text { Uribe } \text { et al. } 2001 \text {, Astudillo et al. } 2009 \\
\text { Uribe } \text { et al. } 2001 \\
\text { Viviani \& DiSalvo } 1980, \text { Von Plessing } 1981 \text {, Uribe } \text { et al. } \\
\text { 2001, Astudillo et al. } 2009 \\
\text { Uribe } \text { et al. } 2001 \\
\text { Uribe } \text { et al. } 2001 \\
\text { Uribe } \text { et al. } 2001 \\
\text { Astudillo et al. } 2009 \\
\text { Viviani \& DiSalvo } 1980, \text { Uribe } \text { et al. } 2001 \\
\text { Astudillo et al. } 2009 \\
\text { Astudillo et al. } 2009 \\
\text { Astudillo et al. } 2009 \\
\text { Astudillo et al. } 2009 \\
\text { Von Plessing } 1981 \\
\text { Astudillo et al. } 2009 \\
\text { Uribe } \text { et al. } 2001 \\
\text { Uribe et al. } 2001 \\
\text { Astudillo et al. } 2009 \\
\text { Astudillo et al. } 2009 \\
\text { Viviani \& DiSalvo } 1980, \text { Astudillo et al. } 2009 \\
\text { Uribe } \text { et al. } 2001 \\
\text { Astudillo et al. } 2009 \\
\text { Astudillo et al. } 2009 \\
\text { Astudillo et al. } 2009\end{array}$ \\
\hline Arthropoda & $\begin{array}{l}\text { Acanthocyclus } \text { sp. } \\
\text { Aeginella sp. } \\
\text { Allopetrolisthes angulosus } \\
\text { Allopetrolisthes spinifrons } \\
\text { Amphipoda } \\
\text { Aora } \text { sp. } \\
\text { Aora typica } \\
\text { Austromegabalanus } \\
\text { psittacus } \\
\text { Balanus amphitrite } \\
\text { Balanus flosculus } \\
\text { Balanus laevis } \\
\text { Bresiliidae } \\
\text { Cancer setosus } \\
\text { Caprella equilibra } \\
\text { Caprella scaura } \\
\text { Caprella verrucosa } \\
\text { Caridea } \\
\text { Corophium } \text { sp. } \\
\text { Deutella venenosa } \\
\text { Elasmopus rapax } \\
\text { Elminius kingii } \\
\text { Ericthonius sp. } \\
\text { Gammaropsis dentifer } \\
\text { Halicarcinus planatus }\end{array}$ & $\begin{array}{l}\text { Sur } \\
\text { Norte } \\
\text { Norte } \\
\text { Norte } \\
\text { Norte/Sur } \\
\text { Norte } \\
\text { Norte } \\
\text { Norte } \\
\text { Norte } \\
\text { Norte/Sur } \\
\text { Norte } \\
\text { Norte } \\
\text { Norte/Sur } \\
\text { Norte } \\
\text { Norte } \\
\text { Norte }\end{array}$ & $\begin{array}{l}\mathrm{S} \\
\mathrm{S} \\
\mathrm{S} \\
\mathrm{M} \\
\mathrm{M} \\
\mathrm{M} \\
\mathrm{M} \\
\mathrm{M} \\
\mathrm{M} \\
\mathrm{M} \\
\mathrm{M} \\
\mathrm{M} \\
\mathrm{S} \\
\mathrm{M} \\
\mathrm{M} \\
\mathrm{M}\end{array}$ & $\begin{array}{c}\mathrm{N} \\
\mathrm{N} \\
\mathrm{N} \\
- \\
\mathrm{N} \\
\mathrm{N} \\
\mathrm{N} \\
\mathrm{N} \\
- \\
- \\
\mathrm{N} \\
\mathrm{N} \\
\mathrm{N} \\
- \\
\mathrm{N} \\
\mathrm{N}\end{array}$ & $\begin{array}{l}\text { Astudillo et al. } 2009 \\
\text { Uribe et al. } 2001 \\
\text { Astudillo et al. } 2009 \\
\text { Uribe et al. } 2001 \text {, Astudillo et al. } 2009 \\
\text { Astudillo et al. } 2009 \text {, presente estudio. } \\
\text { Astudillo et al. } 2009 \\
\text { Uribe et al. } 2001 \\
\text { Viviani \& DiSalvo 1980, Rebolledo 1998, Uribe et al. } \\
\text { 2001, Valdivia et al. } 2005 \text {, Astudillo et al. } 2009 \\
\text { Viviani \& DiSalvo } 1980 \\
\text { Astudillo et al. } 2009 \\
\text { Viviani \& DiSalvo } 1980, \text { Astudillo et al. } 2009 \\
\text { Astudillo et al. } 2009 \\
\text { Von Plessing } 1981, \text { Uribe et al. } 2001, \text { Astudillo et al. } 2009 \\
\text { Astudillo et al. } 2009 \\
\text { Astudillo et al. } 2009 \\
\text { Astudillo et al. } 2009 \\
\text { Astudillo et al. } 2009 \\
\text { Viviani \& DiSalvo } 1980 \text {, Von Plessing } 1981 \\
\text { Astudillo et al. } 2009 \\
\text { Astudillo et al. } 2009 \\
\text { Viviani \& DiSalvo } 1980 \text {, Von Plessing } 1981 \\
\text { Astudillo et al. } 2009 \\
\text { Uribe et al. } 2001 \\
\text { Astudillo et al. } 2009\end{array}$ \\
\hline
\end{tabular}




\begin{tabular}{|c|c|c|c|c|c|}
\hline & Hippolyte sp. & Norte & M & - & Astudillo et al. 2009 \\
\hline & Hyale maroubrae & Norte & M & $\mathrm{N}$ & Uribe et al. 2001 \\
\hline & Hyale media & Norte & M & $\mathrm{N}$ & Astudillo et al. 2009 \\
\hline & Ischycerues sp. & Norte & M & - & Uribe et al. 2001 \\
\hline & Isopoda & Norte & M & - & Astudillo et al. 2009 , presente estudio. \\
\hline & Jassa mamorata & Norte & M & $\mathrm{N}$ & Astudillo et al. 2009 \\
\hline & Jassa slatteryi & Norte & M & $\mathrm{N}$ & Astudillo et al. 2009 \\
\hline & Jassa sp. & Norte & M & - & Uribe et al. 2001 \\
\hline & Latreutes antiborealis & Norte & M & $\mathrm{N}$ & Astudillo et al. 2009 \\
\hline & Lepas anatifera & Norte & $\mathrm{S}$ & $\mathrm{N}$ & Valdivia et al. 2005, Astudillo et al. 2009 \\
\hline & Lepas sp. & Norte & $\mathrm{S}$ & - & Uribe et al. 2001, Astudillo et al. 2009 \\
\hline & Lepas australis & Norte & $\mathrm{S}$ & $\mathrm{N}$ & Astudillo et al. 2009 \\
\hline & Lepas pectinata & Norte & $\mathrm{S}$ & $\mathrm{N}$ & Astudillo et al. 2009 \\
\hline & Leptodius tridentatus & Norte & M & $\mathrm{N}$ & Astudillo et al. 2009 \\
\hline & Liopetrolisthes mitra & Norte & M & $\mathrm{N}$ & Astudillo et al. 2009 \\
\hline & Lysmata sp. & Norte & M & - & Astudillo et al. 2009 \\
\hline & Pachycheles sp. & Norte & M & - & Astudillo et al. 2009 \\
\hline & Paracaprella pusilla & Norte & M & $\mathrm{N}$ & Astudillo et al. 2009 \\
\hline & Paradexamine pacifica & Norte & M & $\mathrm{N}$ & Astudillo et al. 2009 \\
\hline & Petrolisthes tuberculosus & Norte & $\mathrm{M}$ & $\mathrm{N}$ & Astudillo et al. 2009 \\
\hline & Pilumnoides perlatus & Norte & M & $\mathrm{N}$ & Uribe et al. 2001, Astudillo et al. 2009 \\
\hline & Pisoides edwardsi & Norte & M & $\mathrm{N}$ & Uribe et al. 2001, Astudillo et al. 2009 \\
\hline & Pleocyemata & Norte & M & - & Astudillo et al. 2009 \\
\hline & Pycnogonida & Norte & M & - & Astudillo et al. 2009 \\
\hline & Stenothoe sp. & Norte & M & - & Uribe et al. 2001, Astudillo et al. 2009 \\
\hline & Synalpheus spinifrons & Norte & M & $\mathrm{N}$ & Astudillo et al. 2009 \\
\hline & Taliepus dentatus & Norte & M & $\mathrm{N}$ & Astudillo et al. 2009 \\
\hline & Tanais sp. & Norte & M & - & Uribe et al. 2001 \\
\hline & Zeuxo marmoratus & Norte & M & $\mathrm{N}$ & Astudillo et al. 2009 \\
\hline Echinodermata & Arbacia spatuligera & Norte & M & $\mathrm{N}$ & Uribe et al. 2001 \\
\hline & Patiria chilensis & Norte & M & $\mathrm{N}$ & Astudillo et al. 2009 \\
\hline & Tetrapygus niger & Norte & M & $\mathrm{N}$ & Uribe et al. 2001, Astudillo et al. 2009 \\
\hline Urochordata: Ascidacea & Aplidium sp. & Norte & $\mathrm{S}$ & - & Valdivia et al. 2005 \\
\hline & Asterocarpa cerea & Norte & $\mathrm{S}$ & $\mathrm{N}$ & Viviani \& DiSalvo 1980 \\
\hline & Asterocarpa humilis & Norte/Sur & $\mathrm{S}$ & I & $\begin{array}{l}\text { Clarke \& Castilla 2000, Castilla et al. 2005, Castilla \& } \\
\text { Neill 2009, Manríquez. obs. pers. }\end{array}$ \\
\hline & Batryllus schlosseri & Norte & $\mathrm{S}$ & $\mathrm{N}$ & Valdivia et al. 2005 \\
\hline & Ciona intestinalis & Norte & $\mathrm{S}$ & I & $\begin{array}{l}\text { Rebolledo 1998, Uribe et al. 2001, Castilla et al. 2005, } \\
\text { Valdivia et al. 2005, Astudillo et al. 2009, Castilla \& Neill } \\
2009\end{array}$ \\
\hline & Cnemidocarpa robinsoni & Sur & $\mathrm{S}$ & $\mathrm{N}$ & Von Plessing 1981 \\
\hline & Corella eumyota & Norte & $\mathrm{S}$ & $\mathrm{N}$ & Viviani \& DiSalvo 1980 \\
\hline & Didemmnium sp. & Sur & $\mathrm{S}$ & - & Viviani \& DiSalvo 1980 \\
\hline & Diplosoma macdonalsi & Sur & $\mathrm{S}$ & $\mathrm{N}$ & Von Plessing 1981 \\
\hline & Diplosoma sp. & Norte & $\mathrm{S}$ & - & Valdivia et al. 2005, Astudillo et al. 2009 \\
\hline & Lissoclinium sp. & Norte & $\mathrm{S}$ & - & Valdivia et al. 2005 \\
\hline & Molgula ficus & Norte/Sur & $\mathrm{S}$ & I & $\begin{array}{l}\text { Clarke \& Castilla 2000, Castilla et al. 2005, Castilla \& } \\
\text { Neill 2009, Manríquez obs. pers. }\end{array}$ \\
\hline & Paramolgula chilensis & Norte/Sur & $\mathrm{S}$ & $\mathrm{N}$ & Viviani \& DiSalvo 1980 \\
\hline & Paramolgula sp. & Norte & $\mathrm{S}$ & - & Ambler \& Cañete 1991 \\
\hline & Pyura chilensis & Norte & $\mathrm{S}$ & $\mathrm{N}$ & $\begin{array}{l}\text { Viviani \& DiSalvo 1980, Rebolledo 1998, Uribe et al. } \\
\text { 2001, Valdivia et al. 2005, Astudillo et al. } 2009\end{array}$ \\
\hline
\end{tabular}


Anexo 3. Especies de invertebrados $(n=100)$ y macroalgas $(n=33)$ registradas sobre sustratos intermareales rocosos en cada uno de los sitios de muestreo ubicados en los bordes continental e insular del canal de Chacao. Para cada sitio se indica la presencia (+) 0 ausencia (-) de cada especie / Species of invertebrates $(n=100)$ and macroalgae $(n=33)$ recorded on the surface of intertidal substrates in each of the sampling sites located in the continental and insular border of the Chacao channel. For each site the presence (+) or absence (-) of each species is indicated

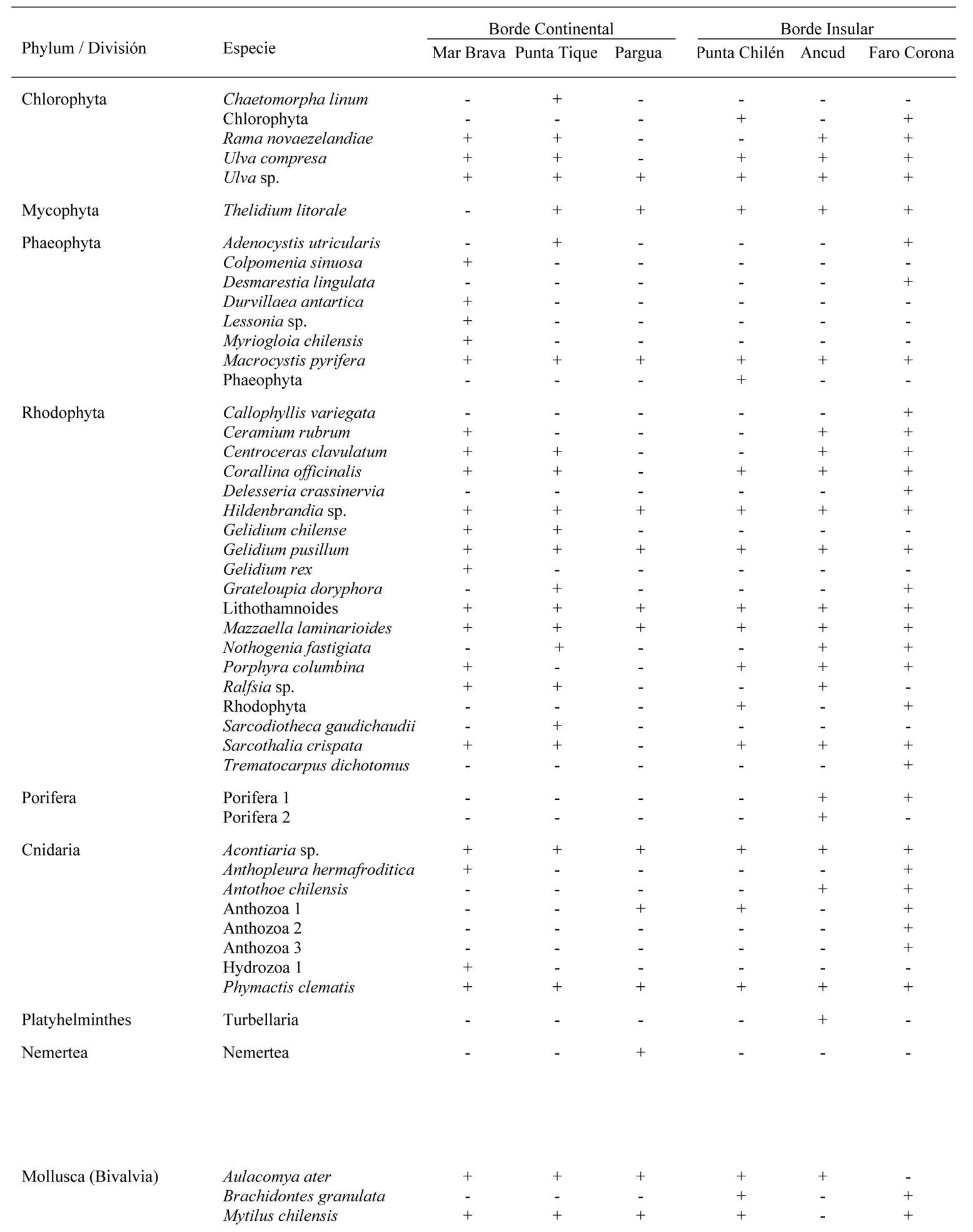


Anexo 3. Continuación / continued

\begin{tabular}{|c|c|c|c|c|c|c|c|}
\hline & Ostrea chilensis & + & - & + & + & + & - \\
\hline & Perumytilus purpuratus & + & + & + & + & + & + \\
\hline & Pholas chiloensis & - & - & - & - & - & + \\
\hline & Semimytilus algosus & - & - & + & - & + & - \\
\hline \multirow[t]{28}{*}{ Mollusca (Gastropoda) } & Acanthina monodon & + & + & + & + & + & + \\
\hline & Austrolittorina araucana & + & + & + & + & + & + \\
\hline & Concholepas concholepas & + & + & - & + & + & + \\
\hline & Crepidula sp. & - & + & - & + & + & - \\
\hline & Diloma nigerrima & + & + & + & + & + & + \\
\hline & Fissurella crassa & - & - & - & - & - & + \\
\hline & Fissurella nigra & + & - & - & + & + & + \\
\hline & Fissurella picta & + & - & - & - & + & + \\
\hline & Fissurella sp. & - & - & + & - & + & - \\
\hline & Lottia orbignyi & - & - & - & + & - & - \\
\hline & Nacella clypeater & + & - & - & - & - & - \\
\hline & Nacella deaurata & - & + & + & - & + & + \\
\hline & Nacella magellanica & + & + & + & + & + & + \\
\hline & Onchidella marginata & + & - & - & - & - & - \\
\hline & Phidiana lottini & - & - & - & + & - & + \\
\hline & Prisogaster niger & + & + & + & + & + & + \\
\hline & Scurria araucana & + & + & + & + & + & + \\
\hline & Scurria bohemita & + & - & - & - & - & - \\
\hline & Scurria ceciliana & + & + & + & + & - & + \\
\hline & Scurria parasitica & + & - & - & - & - & - \\
\hline & Scurria plana & - & - & - & - & + & + \\
\hline & Scurria scurra & + & - & - & - & - & - \\
\hline & Scurria sp. & - & - & - & + & + & - \\
\hline & Scurria variabilis & - & - & + & + & + & + \\
\hline & Scurria zebrina & + & + & + & + & + & + \\
\hline & Siphonaria lessoni & + & + & + & + & + & + \\
\hline & Tegula atra & + & + & + & + & + & + \\
\hline & Xanthochorus cassidiformis & - & + & - & - & - & - \\
\hline \multirow[t]{8}{*}{ Mollusca (Polyplacophora) } & Acanthopleura equinata & - & - & - & - & + & - \\
\hline & Chaetopleura peruviana & - & - & - & - & + & + \\
\hline & Chiton cumingsi & - & - & - & - & - & + \\
\hline & Chiton granosus & + & - & + & + & + & + \\
\hline & Chiton magnificus & + & - & - & + & + & + \\
\hline & Ischnochiton stramineus & - & - & - & - & - & + \\
\hline & Plaxiphora aurata & - & - & - & - & - & + \\
\hline & Tonicia chilensis & - & - & - & - & + & + \\
\hline Mollusca (Cephalopoda) & Octopodidae & - & - & - & - & - & + \\
\hline \multirow[t]{14}{*}{ Annelida (Polychaeta) } & Chaetopteridae & - & - & - & + & + & - \\
\hline & Cirratulus cirratus & - & + & - & - & - & + \\
\hline & Glyceridae & + & - & - & + & - & - \\
\hline & Hemipodus sp. & - & - & + & - & - & + \\
\hline & Hemipodus simplex & - & - & + & - & - & - \\
\hline & Lumbrinereis sp. & - & - & + & - & - & - \\
\hline & Marphysa aenea & - & - & - & - & + & - \\
\hline & Nereididae 1 & - & - & - & + & - & + \\
\hline & Nereididae 2 & - & - & - & + & - & + \\
\hline & Nereis sp. & - & + & + & - & - & - \\
\hline & Perinereis vallata & - & - & - & + & - & - \\
\hline & Polychaeta 1 & + & + & - & - & - & - \\
\hline & Polychaeta 2 & - & + & - & - & - & - \\
\hline & Serpulidae 1 & - & - & - & - & + & + \\
\hline \multirow[t]{3}{*}{ Arthropoda (Crustacea) } & Acantocyclus albatrosis & - & - & - & + & - & - \\
\hline & Amphipoda & + & + & + & + & + & + \\
\hline & Austromegabalanus psittacus & + & - & + & + & + & + \\
\hline
\end{tabular}

264 Manríquez et al.

Bio-incrustantes marinos presentes en el canal de Chacao 
Anexo 3. Continuación / continued

\begin{tabular}{|c|c|c|c|c|c|c|c|}
\hline & & & & & & & \\
\hline & Balanus leavis & + & - & - & - & - & - \\
\hline & Cancer setosus & - & - & - & - & - & + \\
\hline & Cyclograpsus cinereus & - & + & + & + & - & - \\
\hline & Homalaspis plana & - & - & - & - & + & + \\
\hline & Isopoda 1 & - & + & + & + & + & + \\
\hline & Isopoda 2 & - & + & - & + & - & + \\
\hline & Isopoda 3 & - & - & - & - & - & + \\
\hline & Jehlius cirratus & + & + & + & + & + & + \\
\hline & Notobalanus flosculus & + & + & - & + & + & + \\
\hline & Notochthamalus scabrosus & + & + & + & + & + & + \\
\hline & Paraxanthus barbiger & - & - & - & - & - & + \\
\hline & Petrolisthes granulosus & - & - & - & - & - & + \\
\hline & Petrolisthes laevigatus & - & - & + & - & + & - \\
\hline & Petrolisthes sp. & - & - & - & + & + & - \\
\hline & Petrolisthes violaceus & + & - & + & + & - & - \\
\hline & Taliepus dentatus & - & - & - & - & + & - \\
\hline Arthropoda (Chelicerata) & Halacaridae & + & - & + & + & + & - \\
\hline Ectoprocta & Bryozoa 1 & + & + & + & - & - & - \\
\hline & Bryozoa 2 & - & - & - & - & - & + \\
\hline & Bryozoa 3 & - & - & - & - & - & + \\
\hline & Bryozoa 4 & - & - & - & - & - & + \\
\hline & Bryozoa 5 & - & - & - & - & - & + \\
\hline & Celleporella hyalina & - & - & - & - & - & + \\
\hline Echinodermata & Loxechinus albus & - & - & + & - & - & - \\
\hline & Meyenaster gelatinosus & - & - & - & - & - & + \\
\hline Chordata (Urochordata) & Pyura chilensis & + & - & - & - & + & + \\
\hline & Total: & 60 & 50 & 45 & 58 & 64 & 85 \\
\hline
\end{tabular}

